\title{
D-RM Builder: A Software Tool for Generating Fast and Accurate Nonlinear Dynamic Reduced Models from High-Fidelity Models
}

\author{
Jinliang $\mathrm{Ma}^{1,2}$, Priyadarshi Mahapatra ${ }^{1,3}$, Stephen E. Zitney ${ }^{1}$, Lorenz T. Biegler ${ }^{4}$, and David C. \\ Miller $^{5}$ \\ ${ }^{1}$ National Energy Technology Laboratory, Morgantown, WV 26507, USA \\ ${ }^{2}$ AECOM, Morgantown, WV 26505, USA \\ ${ }^{3}$ West Virginia University Research Corporation, Morgantown, WV 26506, USA \\ ${ }^{4}$ Carnegie Mellon University, Department of Chemical Engineering, Pittsburgh, PA 15289, USA \\ ${ }^{5}$ National Energy Technology Laboratory, Pittsburgh, PA 15236, USA
}

\begin{abstract}
Dynamic reduced models (D-RMs) derived from rigorous models are highly desired for speeding up dynamic simulations. A useful software tool named D-RM Builder was developed to automatically generate data-driven D-RMs from high-fidelity dynamic models. It allows a user to configure input/output variables, sample input space and generate sequences of step changes, launch high-fidelity model simulations, fit simulation results to a D-RM, and finally visualize and validate the D-RM. The Decoupled A-B Net (DABNet) nonlinear system identification model was used as the main D-RM type and was enhanced to model nonlinear multiple input and multiple output dynamic systems with options for double-pole formulation to handle fast/slow time scales and pole value optimization. The D-RM Builder tool has been successfully used to generate $\mathrm{D}$-RMs for a highly nonlinear $\mathrm{pH}$ neutralization reactor system and a two-time-scale bubbling fluidized bed adsorber-reactor for $\mathrm{CO}_{2}$ capture.
\end{abstract}

Keywords: Data-driven dynamic reduced models, Nonlinear system identification models, Dynamic simulation, Model predictive control, Engineering software development, Carbon capture

\section{Introduction}

The development, scale-up, and commercialization of an innovative process such as a power generation system with carbon capture has historically required multiple steps ranging from laboratory prototype to pilot scale to demonstration and, finally, to large-scale deployment. Computer simulations have been playing a more important role in optimizing designs of individual devices and entire integrated systems. With increased confidence in computational models, some intermediate steps that are traditionally required in scaling up innovative processes, could potentially be shortened, thereby reducing the time, resources, and cost needed for process commercialization. Modern simulation models cover a wide range of length and time scales, from molecular- to device- to system-scales. The multiscale modeling challenges for carbon capture simulations have been discussed by Miller et al. (2014a). Model reductions 
are sometimes needed to bridge the gap between scales such that a fast, small-scale model can be used in a large-scale model without sacrificing the speed of the simulation (Agarwal et al., 2014). In the time domain, steady-state models are used to optimize process designs while dynamic models are used to optimize process operations such as the transient response of the process in the presence of disturbances. Dynamic process models based on first principles of single or multiple unit operations require the solution of complex systems of differential algebraic equations (DAEs) involving thousands to hundreds of thousands of state variables. Typically, such DAE-based dynamic models are computationally expensive, particularly if the high-fidelity model contains a large number of stiff equations. Also, it is sometimes impractical to apply the high-fidelity models directly in dynamic simulations for off-line operator training systems (OTS) and on-line implementations of advanced process control (APC) and real-time optimization (RTO). For such applications, a fast and accurate dynamic reduced model (D-RM) must often be generated from the rigorous dynamic model.

Two major approaches exist for generating a dynamic reduced model from a DAE-based dynamic model. The first approach involves the simplification of the DAEs in the original highfidelity model and the transformation of the model into projected space, resulting in a new set of DAEs with fewer unknowns and hence faster solution times (Yu et al., 2015). This type of D$\mathrm{RM}$ is known as a reduced-order D-RM with order referring to the number of dynamic variables to be solved. The second approach treats the original rigorous model as a black box model. The transient relationship between the input and output variables of the system is obtained by using results from repeated simulation of multiple step changes in controlled input variables to fit a plant identification model using methods such as nonlinear regression and artificial neural networks (Sjöberg et al., 1995). A D-RM obtained in this way is known as a data-driven D-RM. While the order reduction approach is usually application specific and requires the detailed knowledge of the DAEs involved, the data-driven D-RMs can be generated automatically through a software tool that integrates with DAE solvers.

This paper discusses the generation of data-driven D-RMs through a software tool named D-RM Builder, developed under the auspices of the U.S. Department of Energy's Carbon Capture Simulation Initiative (CCSI) (Miller et al., 2015). The goal of CCSI is to develop models and software tools to help accelerate the scale-up and commercialization of carbon capture technologies. D-RM Builder is a module within the CCSI Framework for Optimization, Quantification of Uncertainty and Surrogates (FOQUS) (Eslick et al., 2014; Miller et al., 2014b). The following sections of the paper describe D-RM Builder's plant identification models, model fitting algorithms, overall software design and structure, typical user workflow, and available features. Finally, two case studies, including a carbon capture case, are provided to illustrate the features and capabilities of D-RM Builder. 


\section{Development of D-RM Builder}

Development of an engineering software application usually involves the implementation of numerical algorithms and the presentation of commands with workflow for human-machine interaction. The development of D-RM Builder follows the same paradigm. First, advanced data-driven D-RMs and their required data structures are identified along with their training algorithms. These nonlinear D-RMs must provide fast and accurate simulations of nonlinear systems for fossil-fired power generation plants with carbon capture. Then, the graphical user interface under a specific development environment is outlined including menus, toolbars, dialog windows, and other widgets through which a user can select parameters and options for model generation, prepare required data, and finally train and validate the D-RM. These commands and dialogs need to be designed such that a user can follow a recommended workflow to generate the D-RM step by step from repeated simulation of the high-fidelity model. In the following sections, the physical models involved are discussed followed by the design and implementation from a software engineering perspective.

\subsection{Data-Driven Dynamic Models}

Plant identification models fall into two major categories: linear and nonlinear. A linear model is used to describe a system consisting of linear ordinary differential equations (ODEs) while a nonlinear model is used for a system consisting of nonlinear ODEs. A classical discretetime state-space-based linear model can be expressed as:

$$
\mathbf{x}(k+1)=\mathbf{A} \mathbf{x}(k)+\mathbf{B u}(k)
$$

Eqn. 1

$$
\mathbf{y}(k)=\mathbf{C x}(k)+\mathbf{D u}(k)
$$

where $\mathbf{x}(k) \in \mathfrak{R}^{n_{x}}$ is the state-space vector of size $n_{x}$ at time $\boldsymbol{k}, \mathbf{u}(k) \in \mathfrak{R}^{n_{u}}$ is the input vector of size $n_{u}$ applied between time $k$ and $k+1$, and $\mathbf{y}(k) \in \mathfrak{R}^{n_{y}}$ is the output vector of size $n_{y}$ at time k. $\mathbf{A}, \mathbf{B}, \mathbf{C}$, and $\mathbf{D}$ in Eqns. 1 and 2 are time-invariant matrices. Commercial software packages such as MATLAB (Mathworks, 2015) have built-in functions to train linear models to find the optimal matrices given a set of transient data. Since most real-world processes such as those found in carbon capture systems are nonlinear, the objective of D-RM Builder is to generate nonlinear D-RMs. There are a variety of nonlinear system identification models in the literature including the Nonlinear Autoregressive Exogenous (NARX) model and the Hammerstein-Wiener model available in MATLAB (Mathworks, 2015). Two types of nonlinear discrete-time models have been implemented in D-RM Builder to date, including classical Nonlinear Auto Regressive Moving Average (NARMA) (Narendra and Mukhopadhyay, 1997) and Decoupled A-B Net (DABNet) (Sentoni et al., 1998). The NARMA model is a special NARX model that uses an artificial neural network (ANN) as the nonlinear function for mapping 
conceptually similar to the Wiener model since both are composed of a linear dynamic block followed by a nonlinear static map. The linear dynamic model of the DABNet is a state-space model based on discrete Laguerre systems while that of the Wiener model is a polynomial transfer function model. The Laguerre model incorporates a crucial process dynamic property the process time-constant information - through "pole" definition, significantly reducing the number of model parameters compared to the Weiner polynomial model and hence is easier to be trained. Furthermore, the static mapping of the DABNet model is a nonlinear mapping from the state-space vector to individual outputs, while that of the Wiener model is a simple scalar-toscalar nonlinear mapping, one for each output variable. This static mapping enables DABNet model to find the nonlinear correlations among multiple state variables within the state vector. The state-space dynamic formulation of the DABNet model makes it easy to implement the correction of the state variables inside a controller through various filtering algorithms such as extended Kalman filter and unscented Kalman filter when the measured output variables are available. The DABNet structure is well suited for embedding into a model predictive control (MPC) strategy, providing better long-term prediction than a NARX model in which the output errors are fed back into the model and amplified (Tsoi and Back, 1994). Since the NARMA model in D-RM Builder is similar to those found in commercial software tools, it will not be discussed further. This paper will focus on the DABNet model and its enhancements implemented in D-RM Builder.

\subsubsection{DABNet Model}

The DABNet model was originally proposed by Sentoni et al. (1998) to approximate nonlinear, causal, discrete time-invariant, multiple-input single-output (MISO) systems with fading memory. It is extended to a multiple-input multiple-output (MIMO) system inside D-RM Builder. Multiple independent DABNet models are used to simulate multiple outputs, one DABNet model for each output. In each DABNet model, linear dynamic mapping from an individual input $u_{j}$ to its corresponding state-space vector $\mathbf{x}_{i j}$ for an output variable $y_{i}$ is modeled by decoupled matrices $\mathbf{A}_{i j}$ and $\mathbf{B}_{i j}$ (one set of matrices for each input-output pair, $j-i$ ) as described in Eqn. 3, where $k$ represents current time and $k+1$ represents the next time step.

$$
\mathbf{x}_{i j}(k+1)=\mathbf{A}_{i j} \mathbf{x}_{i j}(k)+\mathbf{B}_{i j} u_{j}(k)
$$

Note that since $u_{j}$ is a scalar, matrix $\mathbf{B}_{i j}$ contains only one column. If there are $n_{u}$ input variables, then there are $n_{u}$ sets of decoupled $\mathbf{A}_{i j}$ and $\mathbf{B}_{i j}$ matrices with $n_{u}$ sets of state vectors that form an augmented state vector $\mathbf{x}_{i}^{\text {aug }}$ as expressed in Eqn. 4. 


$$
\mathbf{x}_{i}^{a u g}=\left[\begin{array}{c}
\mathbf{x}_{i 1} \\
\mathbf{x}_{i 2} \\
\vdots \\
\mathbf{x}_{i n_{u}}
\end{array}\right]
$$

2 The nonlinear static mapping from the augmented state-space vector $\mathbf{x}_{i}^{\text {aug }}$ to an output variable $3 y_{i}$ is modeled through a feedforward ANN with one hidden layer as described in Eqn. 5:

$$
y_{i}(k)=f_{i}^{A N N}\left(\mathbf{x}_{i}^{\text {aug }}(k)\right)
$$

5 where $f_{i}^{A N N}$ represents the nonlinear function of the ANN for the output $i$.

The linear dynamic system with $\mathbf{A}_{i j}$ and $\mathbf{B}_{i j}$ matrices is initially spanned by a set of 7 discrete Laguerre systems (Sentoni et al., 1998). Figure 1 illustrates the discrete-time transfer 8 function for an input-output pair $j-i$, where $a_{i j}$ and $n_{i j}$ are the pole and the order of the discrete 9 Laguerre system for the input-output pair, respectively. According to Zervos et al. (1988), the 10 pole value $a_{i j}$ should match the main time constant $\tau_{i j}$ and sampling time interval $\Delta t$ as shown 11 in Eqn. 6.

$$
a_{i j}=1-\frac{\Delta t}{\tau_{i j}}
$$

13 The state-space vector for the pair $\mathbf{x}_{i j}$ is a collection of output signals after individual Laguerre 14 blocks (see Figure 1). If a delay in the length of a sampling interval exists, a transfer function of $151 / z$ can be added before the first Laguerre block, making the size of the state-space vector one 16 state longer. 


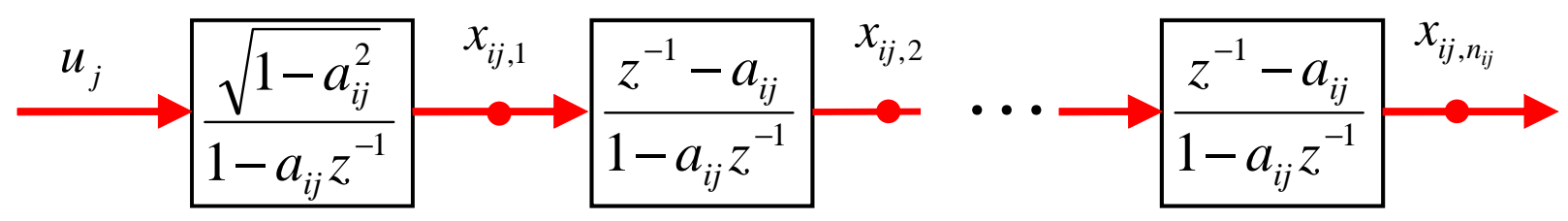

$$
\mathbf{x}_{i j}=\left[\begin{array}{c}
x_{i j, 1} \\
x_{i j, 2} \\
\vdots \\
x_{i j, n_{i j}}
\end{array}\right]
$$

Figure 1. State-space variables for each input-output pair.

3 Given a Laguerre order $n_{i j}$ and pole $a_{i j}$, the decoupled matrices $\mathbf{A}_{i j}$ and $\mathbf{B}_{i j}$ can be readily 4 obtained (see, for example, p. 65 of Zhou et al., 1996). If transient time series data are given for $5 \mathbf{u}(k)$ and $\mathbf{y}(k)$ where $k=0,1,2 \cdots n_{k}$ and the first data point corresponds to a steady-state 6 condition, the initial state-space vector for an input-output pair can be calculated based on Eqn. 7 7:

$$
\mathbf{x}_{i j}(1)=\mathbf{x}_{i j}(0)=\mathbf{A}_{i j} \mathbf{x}_{i j}(0)+\mathbf{B}_{i j} u_{j}(0)
$$

9 or Eqn. 8:

$$
\mathbf{x}_{i j}(0)=\left[\mathbf{I}-\mathbf{A}_{i j}\right]^{-1} \mathbf{B}_{i j} u_{j}(0)
$$

With the initial state vector determined, subsequent state variables at each time step can be calculated based on Eqn. 3. Using the augmented state vector $\mathbf{x}_{i}^{\text {aug }}$ at each time step, the output variable $y_{i}$ at each time step can be calculated by Eqn. 5 if the structure and the weights of neuron connections of the ANN are given.

The weights inside the ANN can then be trained to match the actual response or, as in this case, to match data from the high-fidelity model. The matrices of the linear dynamic model initially obtained from the Laguerre series of finite terms $\left(\mathbf{A}_{i j}\right.$ and $\left.\mathbf{B}_{i j}\right)$ can be reduced by balanced realization as outlined in Sentoni et al. (1998) using the weights between the input and the first hidden layer of the ANN. The reduction procedure is briefly described below.

While Eqn. 5 shows the nonlinear ANN function to map the state vectors $\mathbf{x}_{i}^{\text {aug }}$ to the system output variable $y_{i}$, the nonlinear sigmoid functions involved in the ANN are actually applied only at the first hidden layer neurons, after the weights between the input layer and the 
1 first hidden layer are multiplied by the input state variables and the resulting products are 2 summed. In other words, before applying the nonlinear sigmoid functions, the sums of the 3 products of the weights and the state variables at the individual neurons in the first hidden layer 4 can be considered as intermediate output variables in the DABNet model structure. The linear 5 dynamic system with the intermediate variable $\mathbf{v}_{i j} \in \mathfrak{R}^{n_{h}}$ as the output vector for the decoupled $j$ $6 \quad i$ input-output pair can be described in Eqns. 9 and 10:

$$
\begin{gathered}
\mathbf{x}_{i j}(k+1)=\mathbf{A}_{i j} \mathbf{x}_{i j}(k)+\mathbf{B}_{i j} u_{j}(k) \\
\mathbf{v}_{i j}(k)=\mathbf{W}_{i j} \mathbf{x}_{i j}(k)
\end{gathered}
$$

where $n_{h}$ is the number of the neurons in the first hidden layer and $\mathbf{W}_{i j}$ is a time-invariant $n_{h}$ by $n_{x, i j}$ matrix consisting of the weights between the input and the first hidden layers of the ANN. Because it is a linear dynamic system, all the theory of linear model reduction can be applied. To reduce the dimension of the state vector $\mathbf{x}_{i j}$ or the size of $\mathbf{A}_{i j}$ and $\mathbf{B}_{i j}$ matrices, the balanced realization of the linear system (Zhou et al., 1996) and the truncation based on the Hankel singular values are applied. The observability Grammian $\mathbf{G}_{o, i j}$ and the controllability Grammian $\mathbf{G}_{c, i j}$ are matrices calculated based on the discrete-time Lyapunov equations as shown in Eqns. 11 and 12 .

$$
\mathbf{G}_{o, i j}-\mathbf{A}_{i j}^{T} \mathbf{G}_{o, i j} \mathbf{A}_{i j}=\mathbf{W}_{i j}^{T} \mathbf{W}_{i j}
$$

$$
\mathbf{G}_{c, i j}-\mathbf{A}_{i j} \mathbf{G}_{c, i j} \mathbf{A}_{i j}^{T}=\mathbf{B}_{i j} \mathbf{B}_{i j}^{T}
$$

A similarity transformation $\mathbf{T}_{i j}$ can be obtained such that the system is in balanced form as shown in Eqn. 13:

$$
\mathbf{G}_{o, i j, b a l}=\mathbf{G}_{c, i j, b a l}=\boldsymbol{\Sigma}_{i j}=\operatorname{diag}\left(\sigma_{i j 1}, \sigma_{i j 2}, \cdots, \sigma_{i j n_{i j}}\right)
$$

where $\sigma_{i j 1} \geq \sigma_{i j 2} \geq \cdots \geq \sigma_{i j n_{i j}}$ are Hankel singular values. The balanced system matrices can be expressed as:

$$
\mathbf{A}_{i j, b a l}=\mathbf{T}_{i j} \mathbf{A}_{i j} \mathbf{T}_{i j}^{-1}
$$

$$
\mathbf{B}_{i j, b a l}=\mathbf{T}_{i j} \mathbf{B}_{i j}
$$

Small Hankel values $\left(\sigma_{i j r} \gg>\sigma_{i j r+1}\right)$ indicate that the system can be reduced to an $r$-order model by truncating the $\mathbf{A}_{i j, b a l}$ to an $r$ by $r$ square matrix $\hat{\mathbf{A}}_{i j}$ and $\mathbf{B}_{i j, b a l}$ to an $r$ by 1 matrix $\hat{\mathbf{B}}_{i j}$. Finally, the balanced nonlinear dynamic model becomes: 


$$
y_{i}(k)=\hat{f}_{i}^{A N N}\left(\hat{\mathbf{x}}_{i}^{\text {aug }}(k)\right)
$$

where $\hat{\mathbf{x}}_{i}^{\text {aug }}$ is the augmented state-space vector for output variable $y_{i}$, formed by combining the state-space vectors $\left(\hat{\mathbf{x}}_{i j}\right)$ from all inputs in a similar way as shown in Eqn. 4 . Using the balanced dynamic model of Eqn. 16, the new ANN function $\hat{f}_{i}^{A N N}$ in Eqn. 17 can be trained. Since the size of the state space is smaller, this balanced model is the final model exported from D-RM Builder.

Most nonlinear processes with a single time scale can be reduced to a DABNet model with a single set of Laguerre series associated with a single pole for each input/output pair as described above. However, for complicated processes with two different time scales (slow and fast), the Laguerre series with a limited number of terms was found to be unable to predict both the fast and slow responses as indicated by Mitsis and Marmarelis (2002). To resolve the fast mode, a short sampling time interval has to be used and a small pole selected to match the fast time scale. A large pole is required to match the slow process. Mitsis and Marmarelis (2002) used two Laguerre filter banks with two different poles coupled with a feedforward network with polynomial activation functions to form a Laguerre-Volterra network to model nonlinear physiological systems with both fast and slow dynamics. Their idea of using two Laguerre state vectors, one with a small pole value to match the fast response and the other with a large pole value, is adopted in D-RM Builder. The state-space vector for an input/output pair is now simply formed by combining the two state-space vectors as shown in Eqn. 18:

$$
\mathbf{x}_{i j}=\left[\begin{array}{c}
x_{i j, 1}^{f a s t} \\
x_{i j, 2}^{f a s t} \\
\vdots \\
x_{i j, n_{f}}^{\text {fast }} \\
x_{i j, 1}^{\text {slow }} \\
x_{i j, 2}^{\text {slow }} \\
\vdots \\
x_{i j, n_{s}}^{s l o w}
\end{array}\right]
$$

22 where $n_{f}$ and $n_{s}$ are the orders of the Laguerre systems for the fast and slow responses, 23 respectively. The corresponding $\mathbf{A}_{i j}$ and $\mathbf{B}_{i j}$ matrices are also formed by combining the fast 24 and slow ones as shown in Eqns. 19 and 20. 


$$
\mathbf{A}_{i j}=\left[\begin{array}{cc}
\mathbf{A}_{i j}^{\text {fast }} & 0 \\
0 & \mathbf{A}_{i j}^{\text {slow }}
\end{array}\right]
$$

$$
\mathbf{B}_{i j}=\left[\begin{array}{c}
b_{i j, 1}^{\text {fast }} \\
b_{i j, 2}^{\text {fast }} \\
\vdots \\
b_{i j, n_{f}}^{\text {fast }} \\
b_{i j, 1}^{\text {slow }} \\
b_{i j, 2}^{\text {slow }} \\
\vdots \\
b_{i j, n_{s}}^{\text {slow }}
\end{array}\right]
$$

3 The double-pole approach provides a different formulation for the $\mathbf{A}_{i j}$ and $\mathbf{B}_{i j}$ matrices. The 4 algorithm for the balanced realization is the same as that for the single-pole formulation. D-RM 5 Builder supports both the single-pole and double-pole formulations. It was found that the 6 double-pole approach is capable of modeling processes with two time scales accurately, which 7 can never occur using the single-pole approach. This is a major enhancement over the original 8 DABNet model.

\subsubsection{Model Parameters and Optimization of Pole Values}

The model parameters in the DABNet model include the order of the discrete Laguerre system (i.e., the number of state variables $n_{i j}$ in Figure 1), the pole value $\left(a_{i j}\right.$ in Figure 1) for each input-output pair $j-i$, and the number of neurons in the hidden layer of the ANN for output $i$. When the double-pole option is selected, there are two pole values for each input-output pair. As reported by Sentoni et al. (1998), the identification process is not very sensitive with respect to the order of the discrete Laguerre system $n_{i j}$. Typically, a Laguerre system of order six (the default input of D-RM Builder) produces a good fit for the D-RM. Note that the final order of the dynamic system is obtained by the balanced realization and truncation. In other words, D$\mathrm{RM}$ Builder is able to remove high order terms for a low order system if a high order $n_{i j}$ is initially specified. The pole values were sometimes found to be insensitive to the accuracy of the D-RM for simple dynamic systems while extremely sensitive for some complicated dynamic systems. To handle the latter case, D-RM Builder provides a derivative-free optimizer for selecting the best pole values around user-specified initial guesses. If the user knows the time constant of the process, Eqn. 6 can be used to calculate the pole value as the initial estimate. A default value of 0.5 is recommended if the user has no information about the time constant. When the double-pole option is selected, the user can also calculate the initial guesses for the first (fast) and the second (slow) poles based on Eqn. 6 if the user knows the two time constants. 
default value of 0.95 is recommended for the second pole if the user has no information about the time constants. Usually the optimum pole values obtained are not sensitive to the initial guesses. The built-in optimization feature is based on Nelder-Mead's simplex method (Nelder and Mead, 1965). The objective function that is minimized is the sum of the squared errors for the ANN function over individual time steps based on the Laguerre system matrices rather than the balanced matrices. The balanced realization and the subsequent ANN training are performed after the poles are optimized. Note that that currently the number of neurons in the hidden layer of an individual ANN is not included in the optimization routine. The optimization of this input parameter remains to be explored in the future.

\subsubsection{Uncertainty Quantification of Generated D-RM}

When training a D-RM, the results of high-fidelity model simulations are assumed to be absolutely correct without any noise. In other words, the numerical errors in solving the DAEs are ignored. The accuracy of a D-RM can be measured by the relative error compared to the high-fidelity model used to generate it. A correlation plot of output variables predicted by the DRM versus high-fidelity model results and the corresponding R-squared value also indicate DRM accuracy. Those accuracy related measures can be applied to both training and validation data. However, they are more important for the validation data. Overfitting can usually be detected if the accuracy measures based on validation data are much worse than those based on training data.

The accuracy of a D-RM can also be measured in terms of uncertainties of state-space variables and measured output variables when the D-RM is used for advanced process control. In the control perspective, the state and output variables of the next time step are predicted based on the state and input variables at the current time. When the next time step is reached, the measured output data that contain measurement noise are fed to the control system, in which the state vector at the new time step predicted by the D-RM is corrected using a filtering algorithm such as a Kalman filter. The uncertainty can be quantified by the covariance matrices of the state and output variables. As a user option for a DABNet model, D-RM Builder can perform uncertainty quantification (UQ) based on an unscented Kalman filter (UKF) algorithm in which the generated DABNet model is used as the predictive model and the measurements of output variables are estimated by imposing white noise with user-defined signal to noise ratio (SNR) on the results of the rigorous model simulation. The UKF procedure is adopted from Hartikainen et al. (2011). For any nonlinear discrete-time dynamic system with Gaussian process noise and Gaussian measurement noise, 
where $\mathbf{x}$ and $\mathbf{y}$ are state and measured output vectors, respectively, $\mathbf{q} \sim N(0, \mathbf{Q})$ is the Gaussian

2 process noise, and $\mathbf{r} \sim N(0, \mathbf{R})$ is the Gaussian measurement noise. For the DABNet model, $\mathbf{x}$ 3 is the collection of $\hat{\mathbf{x}}_{i}^{\text {aug }}$ in Eqn. 17 for all output variables $\left(i=1,2, \cdots, n_{y}\right)$, function $f$ in Eqn.

421 consists of multiple linear functions of Eqn. 17 decoupled among multiple input-output pairs, 5 and function $h$ in Eqn. 22 consists of multiple decoupled ANNs of Eqn. 17 for multiple output 6 variables. In D-RM Builder, $\mathbf{Q}$ and $\mathbf{R}$ are diagonal matrices specified by the user for process 7 (state variables) and measurement noises, respectively. Each diagonal element of $\mathbf{R}$ is related to 8 the SNR (in terms of the ratio of signal variance to noise variance) of the corresponding 9 measurement device. The UKF algorithm contains two calculation steps at each time step; one is 10 a prediction step, and the other is an update step. UQ is performed on the transient high-fidelity 11 model results for the validation sequence only. Since the first data point in the transient data 12 represents a steady-state condition, the initial state-space vector $\mathbf{x}(0)$ can be calculated based on 13 an equation similar to Eqn. 8 for the balanced model. Initially a small amount of process noise 14 for state variables represented by a covariance matrix $\mathbf{P}_{o}$ is estimated. In the prediction step, 15 unscented transformation (UT) is applied to the mapping of the current state vector to the state vector of the next time step. $2 n_{x}+1$ sigma points are created where $n_{x}$ is the size of the state vector. Then decoupled linear dynamic functions are evaluated for individual sigma points and the mean $\mathbf{x}^{-}$and covariance matrix $\mathbf{P}^{*}$ of the state vector at next time steps are calculated. The noise matrix $\mathbf{Q}$ is added to the covariance matrix $\mathbf{P}^{*}$ as the predicted process noise matrix $\mathbf{P}^{-}$. The measured output at the new step is assumed to be the results of the high-fidelity model plus a randomly sampled measurement noise from a normal distribution $N(0, \mathbf{Q})$. In the update step, UT is applied to the mapping of the new state vector to the new output vector. $2 n_{x}+1$ sigma points based on predicted noise matrix $\mathbf{P}^{-}$is created and their corresponding outputs are calculated based on Eqn. 17. Then the mean $\mathbf{y}^{-}$and covariance matrix $\mathbf{S}^{*}$ of the output vector are calculated. The measurement noise matrix $\mathbf{R}$ is added to the covariance matrix $\mathbf{S}^{*}$ as the new output noise covariance matrix $\mathbf{S}$. Then cross-covariance matrix $\mathbf{C}$ is calculated from the sigma points and the corresponding Kalman filter gain matrix $\mathbf{K}$ is calculated. Finally the mean of state vector $\mathbf{x}^{-}$is updated or corrected based on the difference between the measured and modeled output $\mathbf{y}-\mathbf{y}^{-}$and the gain matrix $\mathbf{K}$ while the covariance matrix $\mathbf{P}$ of state vector for the next step is updated from the predicted noise matrix $\mathbf{P}^{-}$. The prediction and update processes continue at each time step until all steps in the transient sequence are completed. Figure 2 shows the flow diagram of the UQ analysis procedure. 


\section{Set $\mathbf{Q}, \mathbf{R}$}

Initialize $\mathbf{x}(0)$ based on

steady state input $\mathbf{u}(0)$

Assign $\mathbf{P}_{\mathbf{0}}$

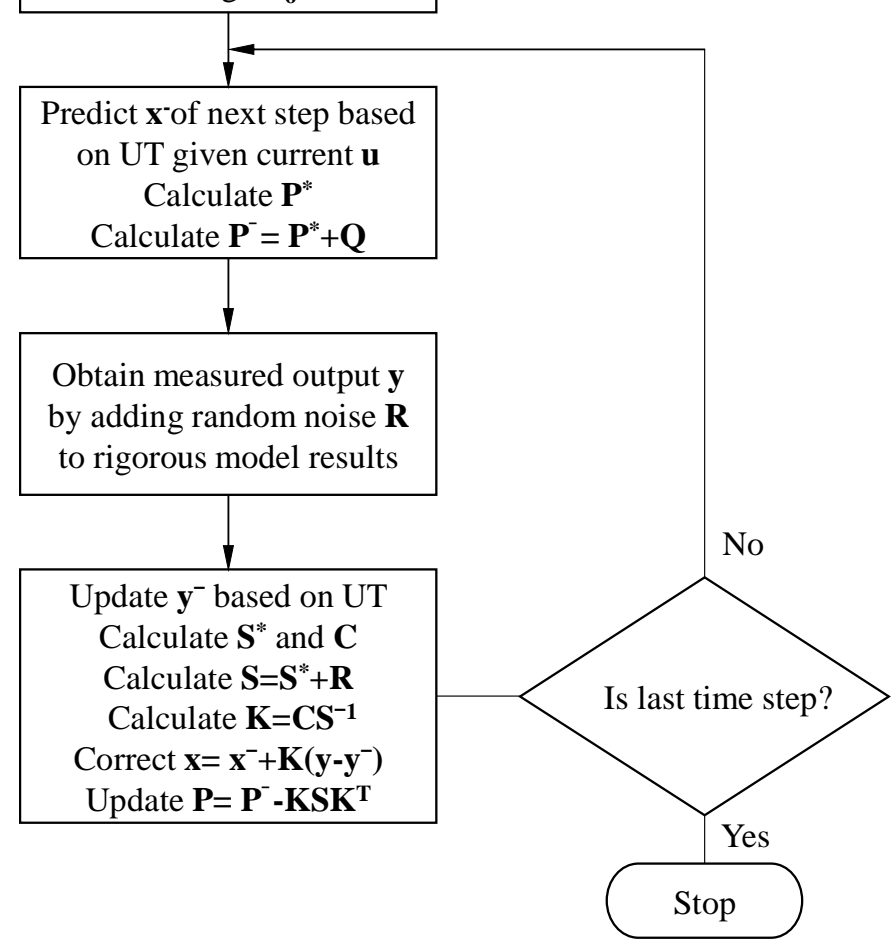

Figure 2. UQ Analysis Procedure

\section{$3 \quad 2.2$ Software Architecture of D-RM Builder}

4 D-RM Builder's architecture is integrated with the existing FOQUS platform and the

5 required algorithms for fitting the transient data to system identification models and for

6 validating the resulting models. FOQUS consists of several modules which enable multiple

7 capabilities including simulation-based process optimization, UQ, optimization under

8 uncertainty, generation of steady-state surrogate model for optimization (Cozad et al., 2014), and

9 D-RM generation. FOQUS has a graphic user interface (GUI) written in Python using open

10 PySide libraries (PySide Project, 2015). The main window of FOQUS contains a set of icons in

11 the upper part and a set of stacked frames. Each icon corresponds to a stacked frame for an

12 individual module. Figure 3 shows the FOQUS GUI with the D-RM Builder module activated; it

13 contains a menu bar, a toolbar, a client area displaying the D-RM as a box connected by input

14 and output variables as arrows and labels, and a client area displaying the messages. 


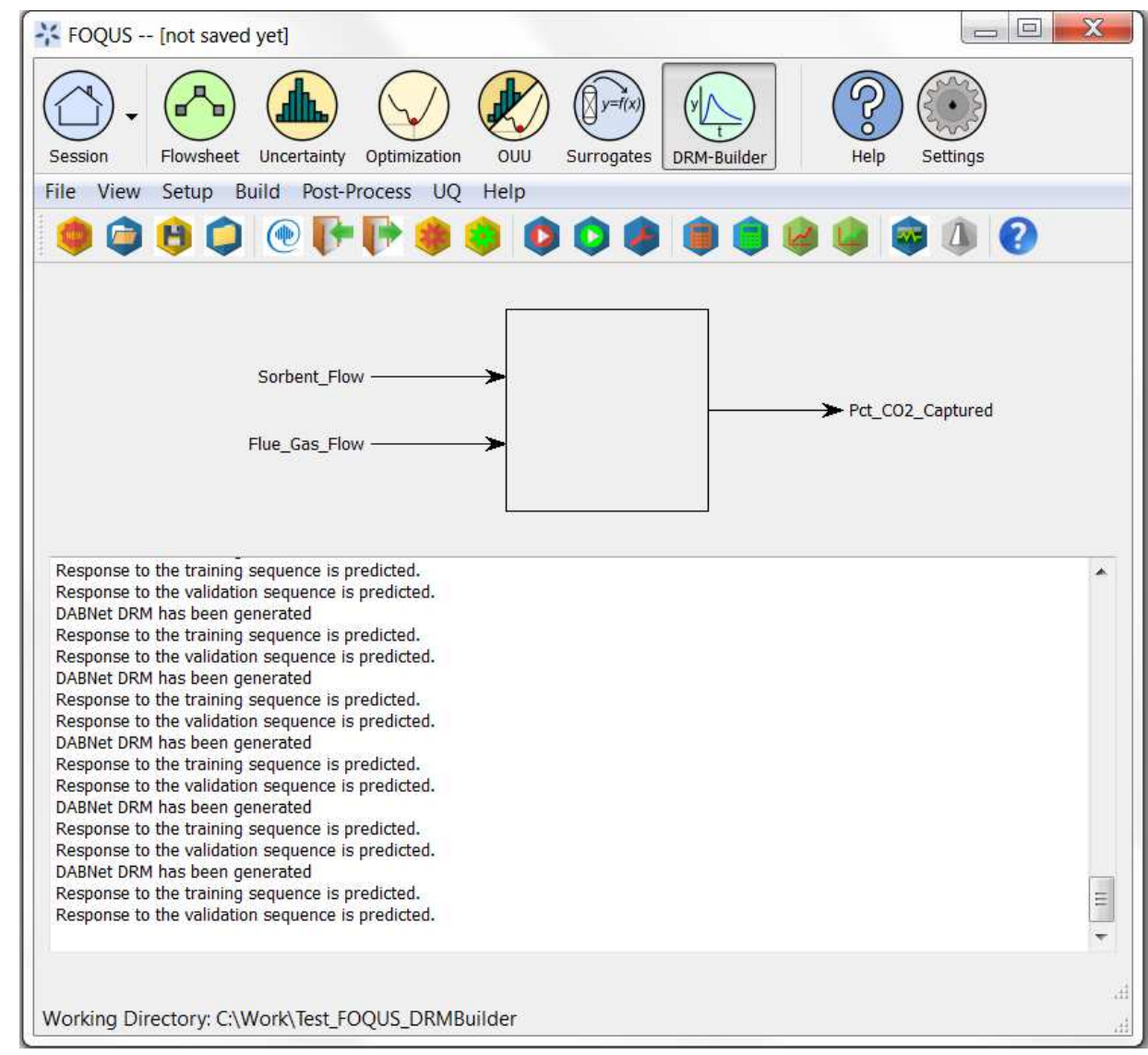

Figure 3. Main window of FOQUS GUI with D-RM Builder activated

Figure 4 shows the workflow of D-RM Builder and the software components used in the process of building a D-RM. D-RM Builder uses three other FOQUS modules, which are shown in the box at the upper left corner of Figure 4. SinterConfigGUI is used to specify the input and output variables of a high-fidelity steady-state or dynamic process model. The high-fidelity dynamic model from which a D-RM is built may contain a large number of input and output variables. Some of the input variables may involve design parameters such as vessel dimensions or certain fixed feed stream data. If the system design and certain operating conditions remain invariant, it is possible that some input variables could be fixed and only a limited number of input variables in the high-fidelity model need to be included in the D-RM. In this paper, the high-fidelity dynamic models are implemented in the commercially available Aspen Custom Modeler (ACM) software package. SinterConfigGUI allows a user to browse ACM variables by querying the ACM model. The user can select a set of input and output (I/O) variables and save them in a file in the open-standard JSON format, which can later be used for scheduling a simulation job. The input variables listed in the JSON file can be assigned values during job execution (i.e., running the process model) and the output variables listed in the JSON file are returned after completion of the simulation. FOQUS provides different ways to run CPUintensive dynamic model simulations via the Turbine module. Turbine also manages the multiple runs needed to build steady-state and dynamic reduced models, perform derivative-free 
optimization or conduct a UQ analysis. Turbine provides the capability for job queuing and enables these jobs to be run in parallel using cloud- or cluster-based computing platforms or a single workstation. A data management framework can store and retrieve simulation files, data and their provenance.

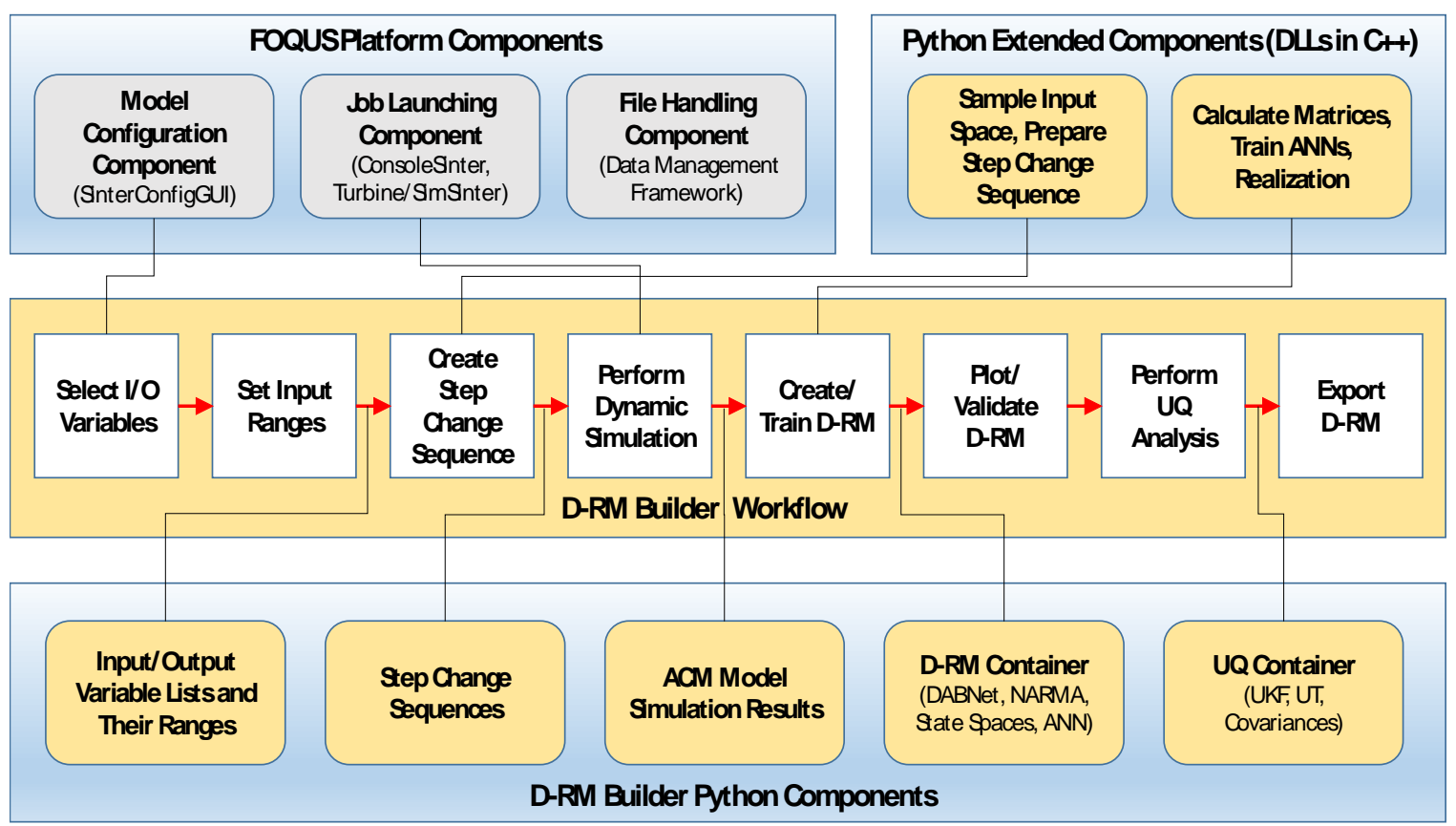

Figure 4. D-RM Builder components and workflow

Generating a D-RM involves several CPU-intensive steps including high-fidelity model simulation, calculating and optimizing D-RM model parameters, and performing UQ analysis. While high-fidelity model simulation is handled by Turbine, some other CPU-intensive calculations need to be coded and compiled in other languages such that they can be run quickly. The two components in the box at the upper right corner of Figure 4 are Python extended components written in $\mathrm{C}++$. They are compiled to dynamically linked libraries (DLLs) in a form that Python can call (with extension "pyd"). The advantage of compiling to a DLL over an executable is that no input and output files are required and all the data are passed in the form of Python tuples. The first Python extended component is for sampling the input space and generating the step change sequence for dynamic model simulation. The second Python extended component is for fitting the training data to a D-RM, including calculating the D-RM parameters and training the ANNs involved. For training ANNs, two types of training algorithms are provided. One is the back propagation (BP) method (Riedmiller, 1994), and the other is the interior point optimization (IPOPT) method (Wächter and Biegler, 2006). Both training options are very CPU-intensive, and there are no open Python libraries available for those algorithms. For the DABNet model, more linear algebra calculations are involved such as 
those for solving Grammian matrices in the balanced realization of the liner system. Therefore, it is better to wrap all D-RM training calculations in a DLL written in C++.

The rest of the D-RM Builder components shown in the bottom box of Figure 4 are written as Python classes, representing I/O variables, step change sequences, transient ACM model simulation data, D-RM related objects (e.g. DABNet model, state-space variables, NARMA model, and ANNs), and UQ related objects (e.g. UKF related data and functions). The Python classes include calculations for predicting the response to a step change sequence using a D-RM in Python's runtime memory. Whenever there is a matrix-related calculation (e.g. in UKF), a fast Python library such as numpy is used. Additional Python classes developed for DRM Builder (not shown in Figure 4) include the GUI classes and some classes to store user inputs. The widgets in the GUI classes are designed using Qt Designer (The Qt Company Ltd., 2015).

As shown in Figure 4, the workflow of D-RM Builder starts when a user selects the desired I/O dynamic variables from a high-fidelity model through SinterConfigGUI. Then the user specifies the ranges (lower and upper limits) of the input variables. The user also specifies the sampling interval for the discrete D-RM model and requests D-RM Builder to create two step change sequences by calling the DLL for step change sequence creation, one for training the DRM and the other for validating the D-RM. The user is allowed to specify the length of the step change sequences by setting the number of Latin hypercube sampling (LHS) points and the duration (in terms of number of sampling time intervals) of holding the input constant before the next step change. Usually the sequence for validation can be much shorter than that for training. The resulting step change sequences can then be passed to Turbine to perform the dynamic simulations. The simulation results are passed back to D-RM Builder. Then the user needs to choose one of the system identification models, either DABNet or NARMA. Several training options such as number of neurons in the hidden layer of ANNs in the D-RM and the training methods (e.g. IPOPT or BP for ANN training) can be specified. The training data and options are passed to another DLL which constructs a Python object representing the D-RM in a D-RM container class. Validating the D-RM consists of predicting the responses of the training and validation sequences using the D-RM and comparing them to the high-fidelity model simulation results. The responses, along with the relative error can be plotted from FOQUS. R-squared values are also displayed as an indication of D-RM accuracy. For the DABNet model, the user is also allowed to specify the noise of the process and measurement and request D-RM Builder to perform UQ analysis on the validation data. If the D-RM is believed to be sufficiently accurate, the D-RM can be exported to a MATLAB script file, which can be used to construct a D-RM object for CCSI's APC Framework. If not, the user can either modify the model parameters or training options to generate a new D-RM or generate additional training data. 


\section{Case Study}

Two example cases are presented in this section to demonstrate the features and 3 capabilities of D-RM Builder. The first case is a continuous-stirred $\mathrm{pH}$ neutralization tank 4 reactor system and the second case is a bubbling fluidized bed (BFB) reactor system for a solid 5 sorbent-based $\mathrm{CO}_{2}$ capture process.

\section{1. pH Neutralization Reactors}

The two-tank $\mathrm{pH}$ neutralization process is a highly nonlinear process that has been widely used as a benchmark dynamic problem in the literature and was also used by the original developers of the DABNet model (Sentoni et al., 1998). Figure 5 shows the schematic of the neutralization system.

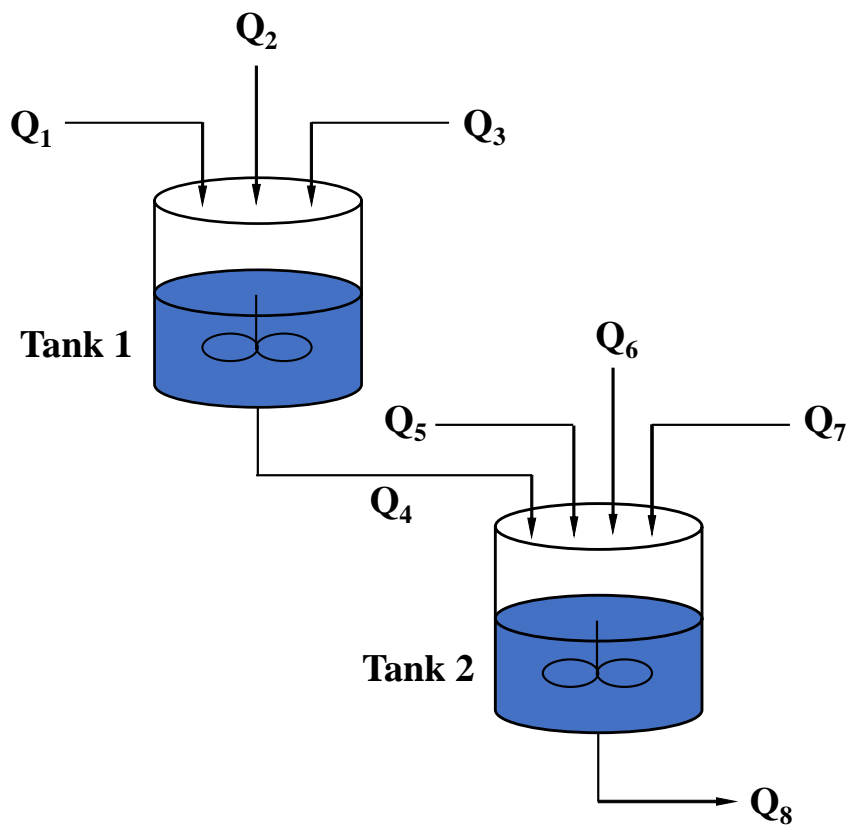

Figure 5. Two-tank pH neutralization system

There are three feed streams to the first tank as shown in Figure 5. $Q_{1}$ and $Q_{2}$ are the volumetric flow rates of acetate acid (HAC) and $Q_{3}$ is the volumetric flow rate of sodium hydroxide $(\mathrm{NaOH})$. The product stream of the first tank with a volumetric flow rate of $Q_{4}$ is fed to the second tank along with three extra feed streams $\left(Q_{5}, Q_{6}\right.$ and $\left.Q_{7}\right) . Q_{5}$ and $Q_{6}$ are the volumetric flow rates of the two HAC streams and $Q_{7}$ is the volumetric flow rate of $\mathrm{NaOH}$.

The corresponding ODEs for the system are listed below:

$$
A_{1} \frac{d h_{1}}{d t}=Q_{1}+Q_{2}+Q_{3}-C_{v 1} \sqrt{h_{1}}
$$




$$
A_{1} h_{1} \frac{d \alpha_{1}}{d t}=Q_{1} C_{A}+Q_{2} C_{A}-C_{v 1} \alpha_{1} \sqrt{h_{1}}
$$

$$
A_{1} h_{1} \frac{d \beta_{1}}{d t}=Q_{3} C_{B}-C_{v 1} \beta_{1} \sqrt{h_{1}}
$$

$$
A_{2} \frac{d h_{2}}{d t}=Q_{5}+Q_{6}+Q_{7}+C_{v 1} \sqrt{h_{1}}-C_{v 2} \sqrt{h_{2}}
$$

$$
A_{2} h_{2} \frac{d \alpha_{2}}{d t}=Q_{5} C_{A}+Q_{6} C_{A}+C_{v 1} \alpha_{1} \sqrt{h_{1}}-C_{v 2} \alpha_{2} \sqrt{h_{2}}
$$

$$
A_{2} h_{2} \frac{d \beta_{2}}{d t}=Q_{7} C_{B}+C_{v 1} \beta_{1} \sqrt{h_{1}}-C_{v 2} \beta_{2} \sqrt{h_{2}}
$$

\section{9}

0

where $\alpha, \beta, A, h$, and $C_{v}$ are acetate $(\mathrm{HAC}+\mathrm{AC})$ concentration, sodium $\left(\mathrm{Na}^{+}\right)$ concentration, cross section area, liquid height, and valve coefficient of a tank, respectively, with the subscript 1 or 2 representing either the first or the second tank. $C_{A}$ and $C_{B}$ are concentration of $\mathrm{HAC}$ and $\mathrm{NaOH}$ in the feed streams, respectively. The algebraic equations related to chemical equilibrium and electroneutrality are listed below:

1

$$
\begin{aligned}
& K_{A}=\frac{\left[A C^{-}\right]\left[H^{+}\right]}{[H A C]} \\
& K_{w}=\left[H^{+}\right]\left[\mathrm{OH}^{-}\right] \\
& \alpha_{2}=[H A C]+\left[A C^{-}\right] \\
& \beta_{2}+\left[H^{+}\right]=\left[O H^{-}\right]+\left[A C^{-}\right] \\
& \mathrm{pH}=-\log _{10}\left[H^{+}\right]
\end{aligned}
$$

Table 1. Normal operating conditions and constants for $\mathrm{pH}$ neutralization example

\begin{tabular}{|l|l|l|l|}
\hline Symbol & Description & Value & Unit of Measurement \\
\hline$A_{1}$ & Cross section area of Tank 1 & 10 & $\mathrm{~m}^{2}$ \\
\hline
\end{tabular}




\begin{tabular}{|l|l|l|l|}
\hline$A_{2}$ & Cross section area of Tank 2 & 10 & $\mathrm{~m}^{2}$ \\
\hline$C_{v 1}$ & Valve coefficient of Tank 1 & 113.1 & $\mathrm{~m}^{2.5} / \mathrm{hr}$ \\
\hline$C_{v 2}$ & Valve coefficient of Tank 2 & 226.2 & $\mathrm{~m}^{2.5} / \mathrm{hr}$ \\
\hline$Q_{1}$ & Acid volumetric flow rate 1 & 2.46 & $\mathrm{~m}^{3} / \mathrm{hr}$ \\
\hline$Q_{2}$ & Acid volumetric flow rate 2 & 2.4 & $\mathrm{~m}^{3} / \mathrm{hr}$ \\
\hline$Q_{3}$ & Base volumetric flow rate 3 & 30.9 & $\mathrm{~m}^{3} / \mathrm{hr}$ \\
\hline$Q_{5}$ & Acid volumetric flow rate 5 & 3.0 & $\mathrm{~m}^{3} / \mathrm{hr}$ \\
\hline$Q_{6}$ & Acid volumetric flow rate 6 & 1.86 & $\mathrm{~m}^{3} / \mathrm{hr}$ \\
\hline$Q_{7}$ & Base volumetric flow rate 7 & 30.9 & $\mathrm{~m}^{3} / \mathrm{hr}$ \\
\hline$C_{A}$ & HAC concentration & 0.32 & $\mathrm{kmol} / \mathrm{m}^{3}$ \\
\hline$C_{B}$ & $\mathrm{~N}_{\mathrm{a}}$ OH concentration & 0.05 & $\mathrm{kmol} / \mathrm{m}^{3}$ \\
\hline$K_{A}$ & HAC dissociation constant & $1.8 \times 10^{-5}$ & \\
\hline$K_{B}$ & $\mathrm{H}_{2}$ O dissociation constant & $1.0 \times 10^{-14}$ & \\
\hline
\end{tabular}

Eqns. 23-33 are coded in an ACM model with six feed streams $\left(Q_{1}, Q_{2}, Q_{3}, Q_{5}, Q_{6}\right.$, and $\left.Q_{7}\right)$ and one final product stream $\left(Q_{8}\right)$. The first step for generating a D-RM for the dynamic system is to configure the I/O variables through SinterConfigGUI. In this example, only two input variables and two output variables are included as the dynamic variables in the DRM while all other input variables are kept as constants at normal operating conditions. The two dynamic input variables are the acid flow rate $Q_{1}$ and the base flow rate $Q_{3}$, both of which are volumetric flow rates of feed streams to Tank 1. The two output variables are the acetate (HAC $+\mathrm{AC}^{-}$) concentration $\alpha_{2}$ and $\mathrm{pH}$ value in the final product stream $Q_{8}$. To generate the D-RM for the $\mathrm{pH}$ neutralization system, a sampling time interval of $0.005 \mathrm{hr}$ is used. The two input dynamic variables are allowed to change within $\pm 10 \%$ of the steady-state values at normal operating conditions as listed in Table 1. In other words, the lower and upper limits of $Q_{1}$ are 2.214 and $2.706 \mathrm{~m}^{3} / \mathrm{hr}$, respectively, and those of $Q_{3}$ are 27.81 and $33.99 \mathrm{~m}^{3} / \mathrm{hr}$, respectively.

To generate a step change sequence for training, five sets of LHS samples are randomly generated by D-RM Builder with each set containing five steady-state points in the 2-D input space. A total of 25 steady-state points are sampled. The step change sequence is obtained by making two step changes to move to a new steady-state point, one for each input variable. The holding times in terms of number of sampling time intervals after each step change are also specified as D-RM Builder input. For this example, the holding durations are 5, 10, 15, 5, and 10 sampling time intervals for the five sets of LHS samples, respectively. Different holding durations are needed to excite the system in a range of frequencies. After switching to 25 steadystate points from the first point to the last point, the process is reversed. Therefore, a total of 
$150 \times 2=100$ step changes are generated with different holding durations. The total time covered 2 by those step changes including the initial holding times before the first step change is $3.52 \mathrm{hr}$ or 3704 sampling time intervals. Plots (a) and (b) in Figure 6 show the step change sequence created 4 by D-RM Builder.

5 DABNet is used as the D-RM type. The options selected for generating the D-RM are 6 listed in Table 2. The BP method is used for training ANNs of both Laguerre and balanced 7 models. The number of neurons in the hidden layer is set to 5 for both output variables $\alpha_{2}$ and $8 \mathrm{pH}$. The number of Laguerre states is set to 6 and 7 for $\mathrm{I} / \mathrm{O}$ pairs involving $\alpha_{2}$ and $p H$, 9 respectively. No time delays are applied for all I/O pairs. Single-pole Laguerre series is used for 10 all I/O pairs. Initially all pole values are set to the default value of 0.5 and are then optimized by 11 D-RM Builder.

12 It took approximately $0.5 \mathrm{hr}$ to perform the ACM simulations to predict the response to 13 the step change sequence for training. It took approximately $180 \mathrm{~s}$ to train the two DABNet 14 models (one for each output) for the $\mathrm{pH}$ neutralization reactor system including the pole 15 optimization. All computations including ACM simulation and D-RM training were conducted 16 on a Dell Precision M3800 (2.2 GHz Intel Core i7) workstation. The required time for training 17 the two DABNet models at a given set of pole values is approximately $6 \mathrm{~s}$, including calculating 18 the $\mathbf{A}_{i j}$ and $\mathbf{B}_{i j}$ matrices of the Laguerre system, training the initial ANN, performing balanced 19 realization to calculate $\hat{\mathbf{A}}_{i j}$ and $\hat{\mathbf{B}}_{i j}$ matrices, and finally retraining the ANN based on the 20 reduced matrices. Approximately 40 to 70 iterations are required to optimize the pole values for 21 each output variable. Note that the balanced realization and the ANN training for the balanced 22 model are performed only once, after the completion of the pole optimization as described in 23 Section 2.1.2. 


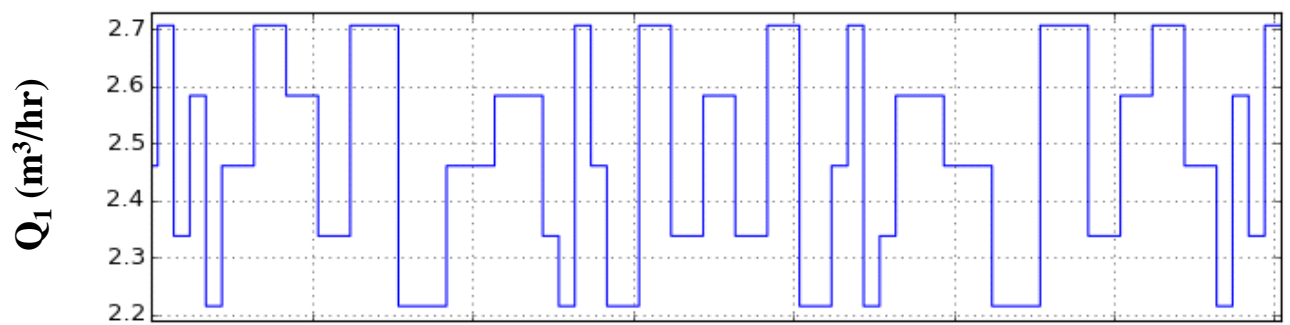

(a)

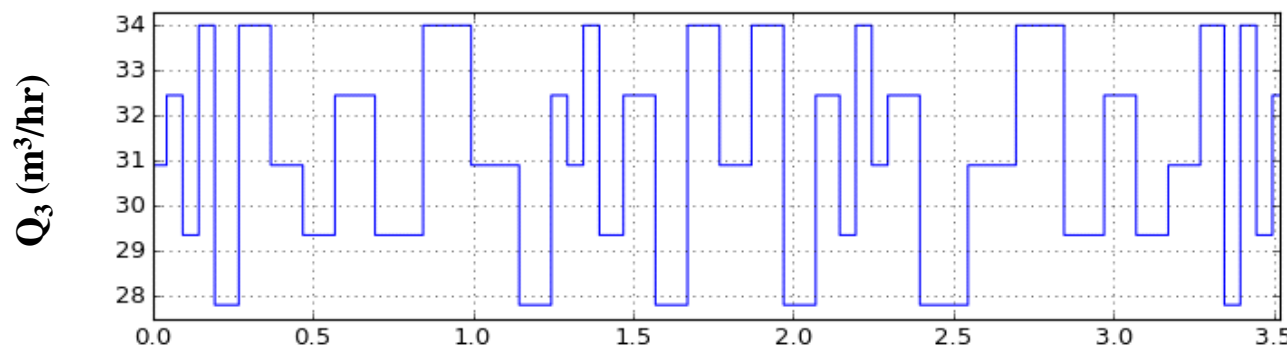

(b)

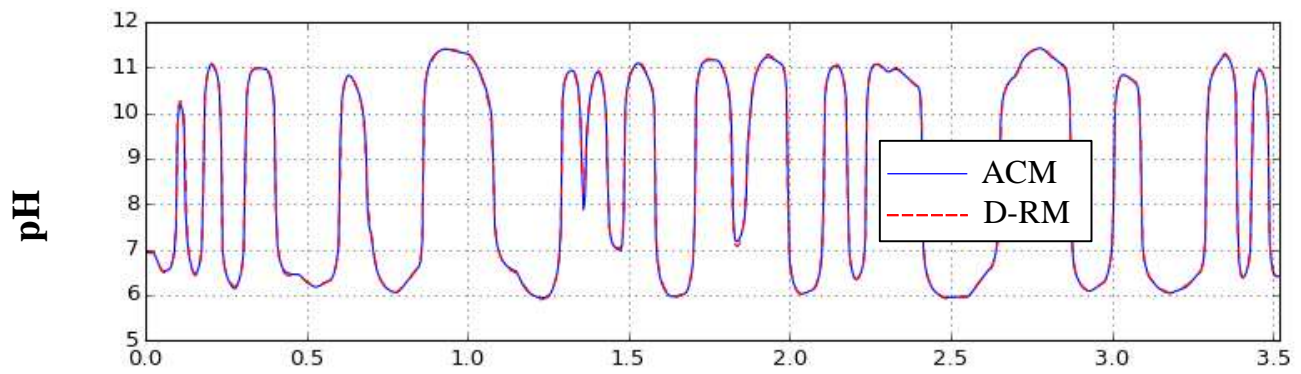

(c)

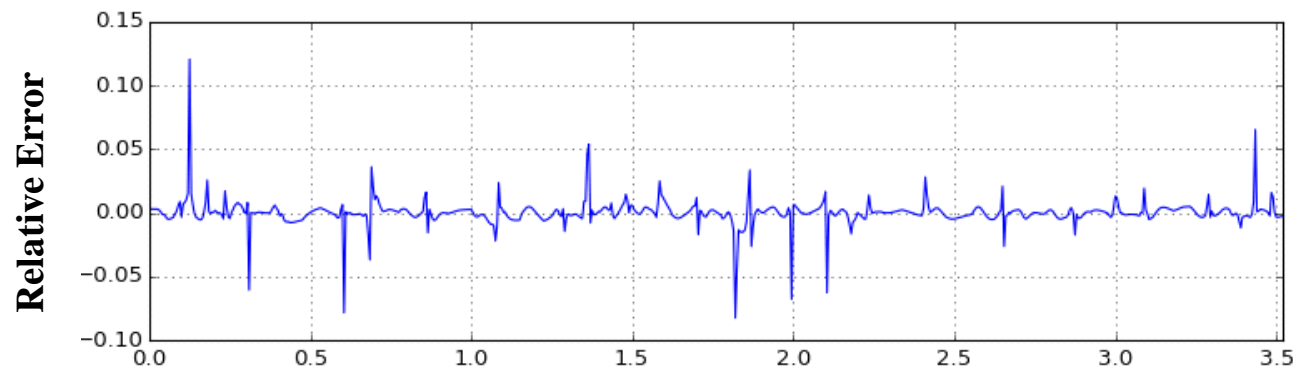

(d)

1

Table 2. Options for training D-RM of $\mathrm{pH}$ neutralization example

\begin{tabular}{|l|c|}
\hline \multicolumn{1}{|c|}{ Option } & Selection \\
\hline Model type & DABNet \\
\hline Method for training Laguerre model ANN & BP \\
\hline
\end{tabular}




\begin{tabular}{|l|c|}
\hline Method for training balanced model ANN & BP \\
\hline Maximum number of iterations for training Laguerre model ANN & 7000 \\
\hline Maximum number of iterations for training balanced model ANN & 10000 \\
\hline Number of neurons in hidden layer for output $\alpha_{2}$ & 5 \\
\hline Number of neurons in hidden layer for output $p H$ & Yes \\
\hline Optimize pole values for output $\alpha_{2}$ & Yes \\
\hline Optimize pole values for output $p H$ & No \\
\hline Time delay applied for I/O pair $Q_{1} / \alpha_{2}$ & 6 \\
\hline Number of Laguerre states for I/O pair $Q_{1} / \alpha_{2}$ & No \\
\hline Time delay applied for I/O pair $Q_{3} / \alpha_{2}$ & 6 \\
\hline Number of Laguerre states for I/O pair $Q_{3} / \alpha_{2}$ & No \\
\hline Time delay applied for I/O pair $Q_{1} / p H$ & 7 \\
\hline Number of Laguerre states for I/O pair $Q_{1} / p H$ & No \\
\hline Time delay applied for I/O pair $Q_{3} / p H$ & 7 \\
\hline Number of Laguerre states for I/O pair $Q_{3} / p H$ & 0.5 \\
\hline Initial pole value for all I/O pairs & \\
\hline
\end{tabular}

After optimization, the pole values used for the D-RM are listed in Table 3. The order of the model (number of state variables) for I/O pairs are reduced through balanced realization as shown in Table 3 . On average, they are reduced by more than $50 \%$ from their initial values. The average ANN training errors $E_{A N N}$ defined in Eqn. 34 for normalized output variables are also listed in Table 3:

$$
E_{A N N}=\frac{\left(y_{t}-y_{o}\right)^{2}}{2}
$$

where $y_{t}$ is the target output value from ACM simulation and $y_{o}$ is the D-RM predicted output value. Note that within an $\mathrm{ANN}$, any output value $y$ is normalized by the mean $\bar{y}$ and the standard deviation $\sigma_{y}$ of all output data points in the training sequence based on Eqn. 35 and the normalized value $\hat{y}$ is actually used in Eqn. 34 to train the ANN.

$$
\hat{y}=\frac{y-\bar{y}}{\sigma_{y}}
$$




\begin{tabular}{|l|c|}
\hline Optimized pole value of balanced model for I/O pair $Q_{1} / \alpha_{2}$ & 0.7794 \\
\hline Optimized pole value of balanced model for I/O pair $Q_{3} / \alpha_{2}$ & 0.8445 \\
\hline Optimized pole value of balanced model for I/O pair $Q_{1} / p H$ & 0.8456 \\
\hline Optimized pole value of balanced model for I/O pair $Q_{3} / p H$ & 0.8644 \\
\hline Number of states of balanced model for I/O pair $Q_{1} / \alpha_{2}$ & 3 \\
\hline Number of states of balanced model for I/O pair $Q_{3} / \alpha_{2}$ & 3 \\
\hline Number of states of balanced model for I/O pair $Q_{1} / p H$ & 4 \\
\hline Number of states of balanced model for I/O pair $Q_{3} / p H$ & 2 \\
\hline Average error of the balanced model ANN for output $\alpha_{2}$ & 0.000105 \\
\hline Average error of the balanced model ANN for output $p H$ & 0.000783 \\
\hline
\end{tabular}

1

2 If the training errors are small, the response calculated by the D-RM should match the response 3 calculated by the ACM simulation. Plot (c) in Figure 6 shows a comparison between the two

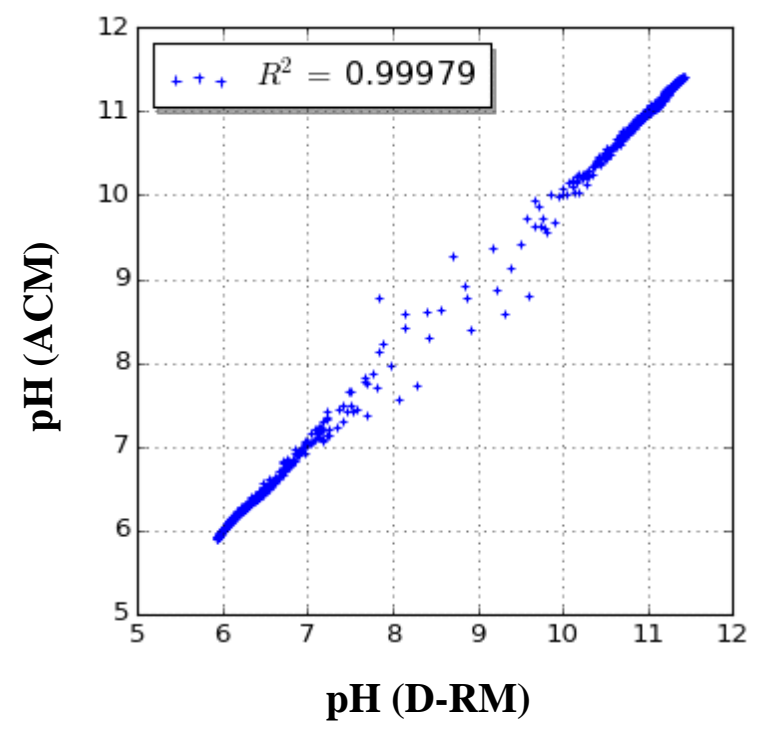


(b) Validation data

Figure 7. Correlation plot of $\mathrm{pH}$ values predicted by ACM and D-RM

3

Since the D-RM is generated by fitting the ACM model results, the D-RM prediction can usually match the ACM prediction quite well. To validate the model, a different step change sequence is generated and the dynamic response to the new step changes is simulated by ACM. The corresponding response is then predicted by the D-RM. The D-RM prediction is compared to the ACM result for the validation sequence in Figure 8, along with the relative errors and step changes. The corresponding correlation plot is shown in Plot (b) of Figure 7. It can be seen from Figure 8 that the D-RM prediction, the red dashed curve in Plot (c), matches the ACM result, the blue solid curve. The R-squared value of 0.99853 for the validation data is also close to unity, indicating a good match.

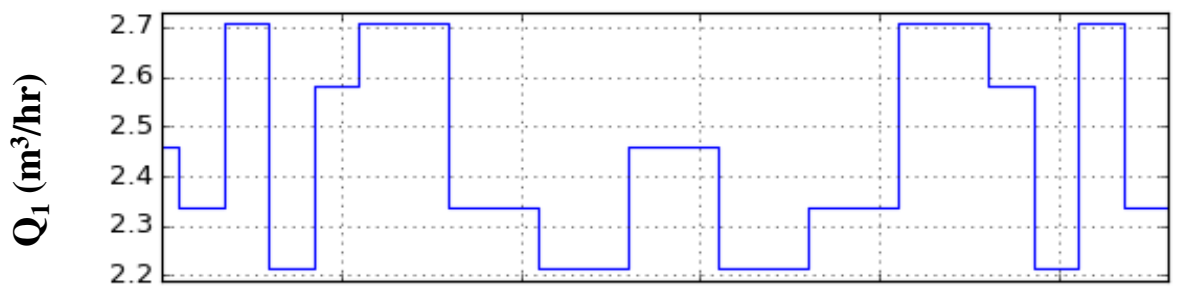

(a)

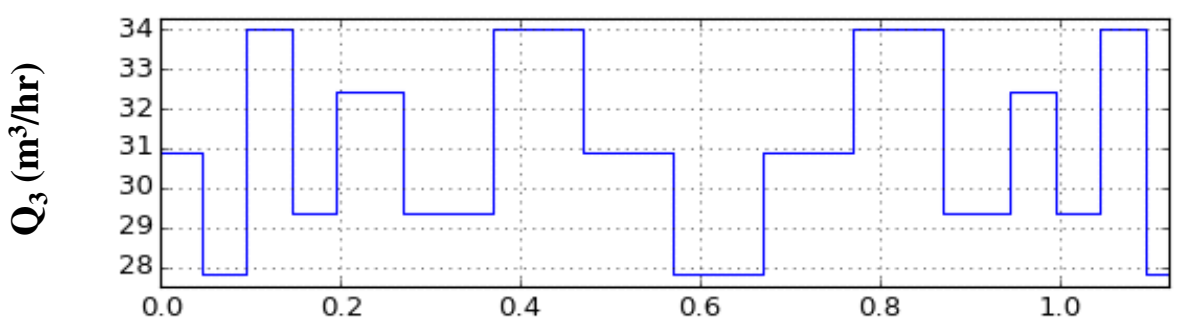

(b)

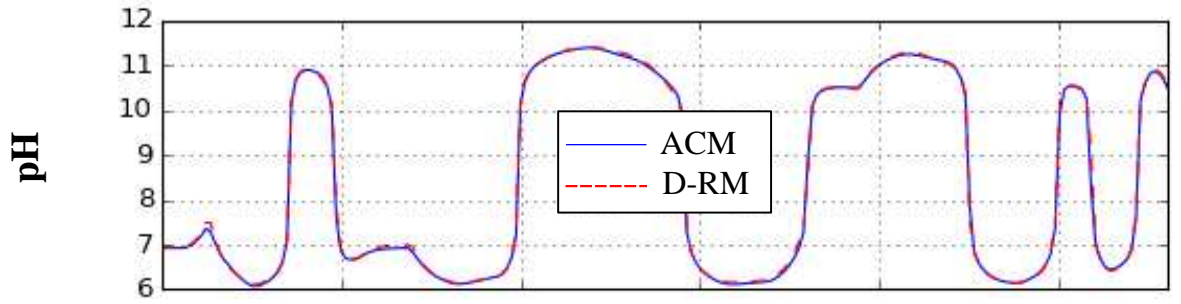

(c)

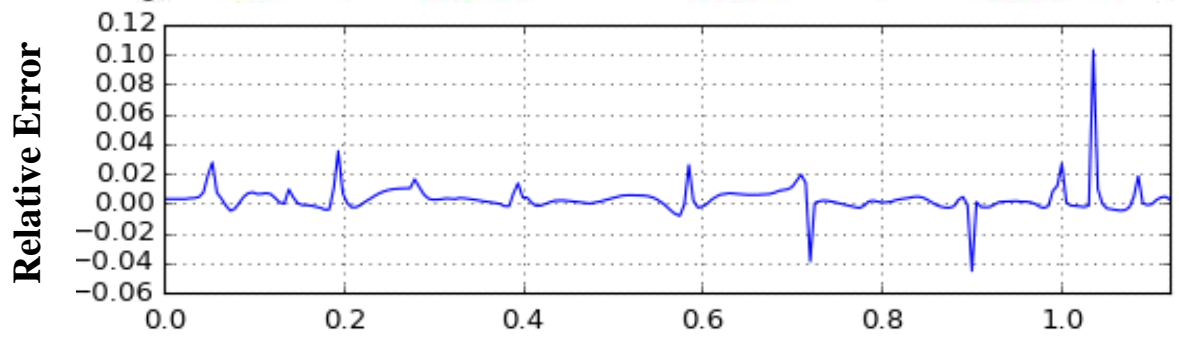

(d)

Time (hr)

(a) Step change sequence of $Q_{1}$

(c) $\mathrm{pH}$ predicted by ACM and D-RM (b) Step change sequence of $Q_{3}$

(d) Relative error of D-RM prediction 
Figure 8. Step change sequences for validating the D-RM of $\mathrm{pH}$ neutralization reactor system and comparison between ACM and D-RM predictions is applied to the validation data with noise specified for state-space and output variables. In this example, the noise for all state-space variables is specified as $1 \%$ of the standard deviation of individual state-space variables based on the training data. The measurement noises for $\mathrm{pH}$ and acetate $\left(\mathrm{HAC}+\mathrm{AC}^{-}\right)$concentration $\alpha_{2}$ in the product stream $Q_{8}$ (see Figure 5) are assumed to be $5 \%$ of the standard deviations of the two output variables from the training data. The covariance matrix of the UQ analysis on the validation data is listed in Table 4. The two diagonal elements of the matrix represent the variances of the filtered output variables, from which their standard deviations can be calculated. The two off-diagonal elements are very small in this case, indicating that the two output variables are not correlated. Figure 9 shows the filtered response curves with a section of the curves in the dashed rectangular region of the upper plot enlarged and displayed in the lower plot. The blue solid curve in Figure 9 is the measured $\mathrm{pH}$ value in the product stream. Notice that the zigzag lines are due to measurement noise. The red dashed curve in Figure 9 is the filtered prediction, which is much smoother. The black dotted curve and black double dotted curve show the range of the filtered result with a standard deviation calculated from the diagonal of covariance matrix $\mathbf{S}$ added to and subtracted from the filtered result (red dashed curve).

Table 4. Final covariance matrix of output variables

\begin{tabular}{|l|c|c|}
\hline & $\alpha_{2}$ of Stream $Q_{8}$ & pH of Stream $Q_{8}$ \\
\hline$\alpha_{2}$ of Stream $Q_{8}$ & $1.7 \times 10^{-9}$ & $-3.46 \times 10^{-18}$ \\
\hline pH of Stream $Q_{8}$ & $-3.46 \times 10^{-18}$ & 0.01151 \\
\hline
\end{tabular}



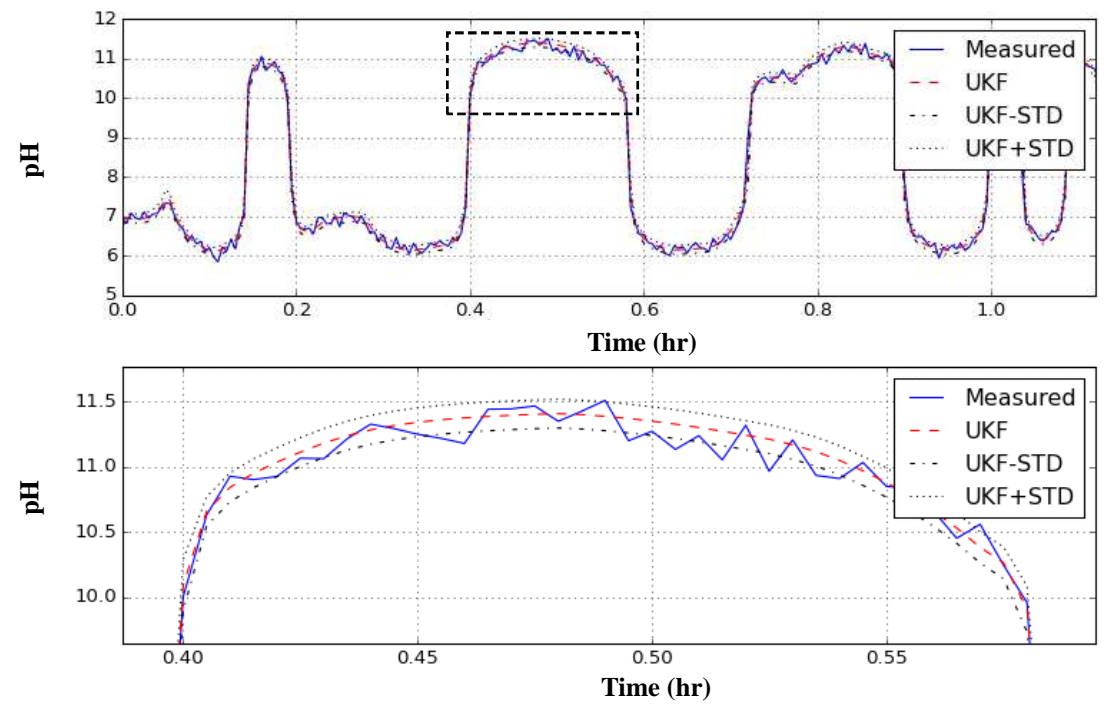

Figure 9. Response of the DABNet model for validation sequence

\section{3}

4

\subsection{Bubbling Fluidized Bed $\mathrm{CO}_{2}$ Adsorber-reactor}

Solid-sorbent-based post-combustion $\mathrm{CO}_{2}$ capture was one of the challenging industry problems considered by CCSI. An ACM model was developed for a bubbling fluidized bed (BFB) adsorber for capturing $\mathrm{CO}_{2}$ from post-combustion flue gas (Lee and Miller, 2013; Modekurti et al., 2013; Mahapatra et al., 2014). The adsorber uses an amine-based solid sorbent, and the adsorber consists of two BFBs in countercurrent series with cooling tubes embedded in each reactor. The process flowsheet in ACM is shown in Figure 10. Detailed information about the ACM model can be found in Modekurti et al. (2013).

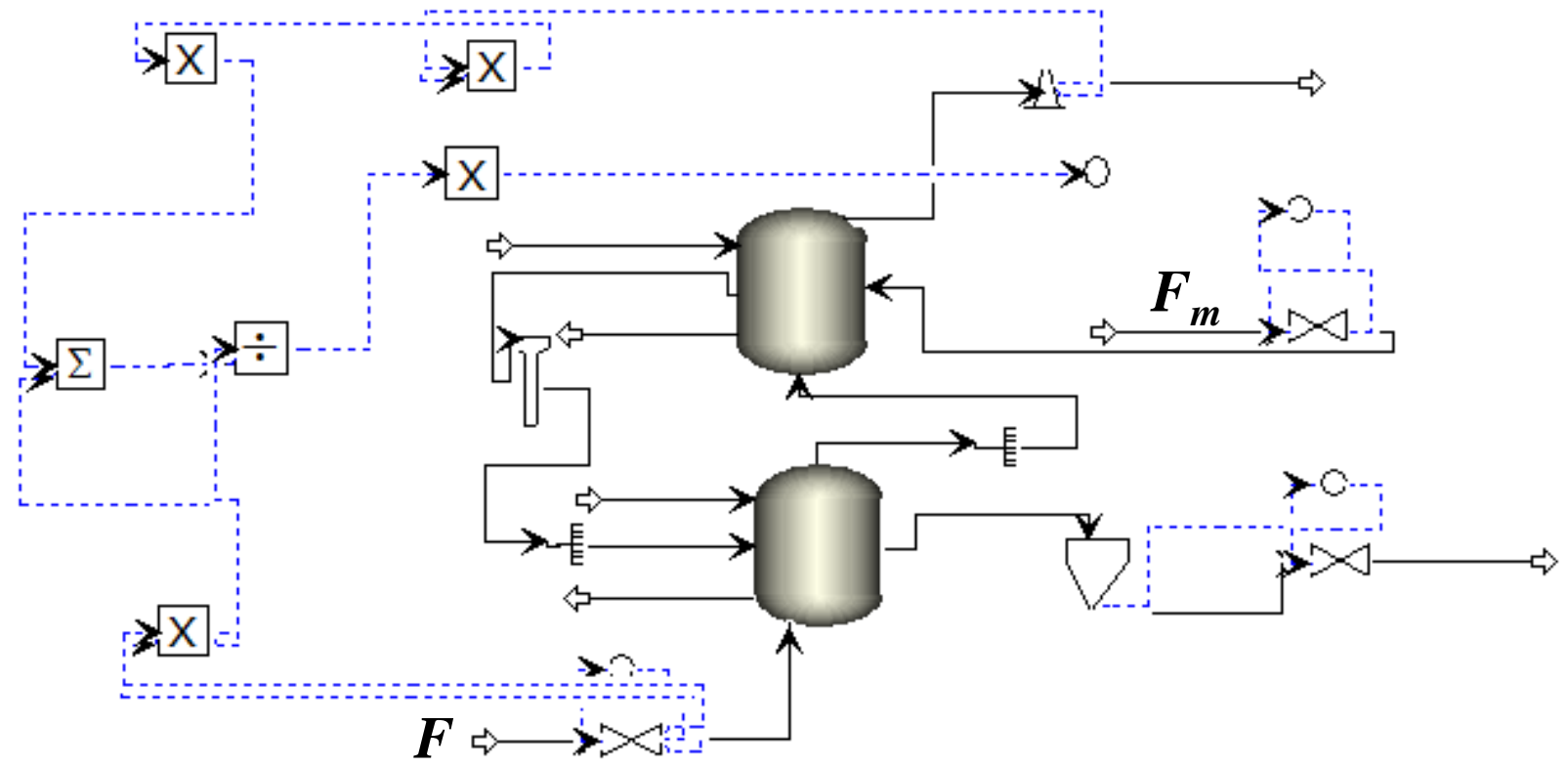


In this case study, a D-RM is generated from the rigorous ACM model with the flow rate of flue gas fed to the system $F$ and the flow rate of the sorbent stream $F_{m}$ as the two input variables and the percentage of $\mathrm{CO}_{2}$ captured as the only output variable. The gas/solid twophase reaction/separation system has both a fast and a slow time scale. The fast time scale is on the order of a few seconds due to the rapid response of fluid dynamics of the two-phase flow while the slow time scale is on the order of hundreds of seconds due to the slow kinetics of the solid sorbent. Table 5 lists the normal operating conditions for the two input variables and their lower and upper limits. Again, $\pm 10 \%$ of the normal operating conditions are defined as the valid range for the D-RM.

Table 5. Range of input variables defined for the D-RM of the BFB adsorber-reactor

\begin{tabular}{|l|c|c|}
\hline Input Variable & Feed Flue Gas Flow Rate $\boldsymbol{F}$ & Feed Sorbent Flow Rate $\boldsymbol{F}_{\boldsymbol{m}}$ \\
\hline Unit of Measurement & $\mathrm{kmol} / \mathrm{hr}$ & $\mathrm{kg} / \mathrm{hr}$ \\
\hline Normal Operating Condition & 6,750 & 600,000 \\
\hline Lower Limit & 6,075 & 540,000 \\
\hline Upper Limit & 7,425 & 660,000 \\
\hline
\end{tabular}

To resolve the fast time scale, a sampling time interval of $1.0 \mathrm{~s}$ is used. To generate the step change sequence for training, four sets of LHS samples are generated, each of which has five steady-state operating conditions randomly selected in the ranges listed in Table 5. The holding times are 20,40,60, and $80 \mathrm{~s}$ for the four sets of samples, respectively. Step changes are formed by marching the individual steady-state condition from the first sample to the last and then from the last to the first (selecting the option with reversed step changes from the GUI of DRM Builder). To validate the D-RM, two sets of samples are generated, each of which has five steady-state operating conditions randomly selected in the same ranges. The holding times are 30 and $70 \mathrm{~s}$ for the two sets of samples, respectively. The option with reversed step changes is also selected. Figure 11 shows the step change sequence for validation. The step change sequences for both training and validation are sent to the ACM model through Turbine. The results of ACM simulations are returned to D-RM Builder for training and validating the D-RM.

Initially a single-pole option of the DABNet model is used to generate the D-RM. For a process with a single time constant around a few seconds (corresponding to the fast time scale of the BFB adsorber-reactor), a sampling time of $1.0 \mathrm{~s}$, a pole value near 0.5 (see Eqn. 6), and a Laguerre order of 6 are usually good enough to train the DABNet model. However, the slow 
1 Laguerre order needs to be used. Note that Laguerre terms at lower orders (the first few blocks 2 in Figure 1) tend to fit a faster response, and those at higher orders tend to fit a slower response. 3 It was initially expected that the long Laguerre series can fit both the fast and slow responses. 4 However, too long a Laguerre series is impractical; it is extremely hard to train the ANN 5 involved. A single pole value with initial guess of 0.8 is optimized for a Laguerre order of 8 for 6 the two I/O pairs. Figure 12 shows the transient profile of the output variable, the percentage of $7 \quad \mathrm{CO}_{2}$ captured, predicted by the D-RM with the single-pole option for the validation sequence, the 8 red dashed curve, and the one calculated by the rigorous ACM model, the blue solid curve. It 9 can be seen from Figure 12 that there is a fast response at every step change followed by a slow 10 response. The fast responses due to the step changes in feed flue gas flow rate are more dramatic 11 than those of the sorbent flow rate. Apparently the optimized single-pole model cannot capture 12 both the fast and slow responses, especially the response due to the change in sorbent flow rate. 13 The average error when training the ANN of the balanced model is $6.35 \times 10^{-4}$ and the $R^{2}$ value 14 for the validation data is 0.993 .
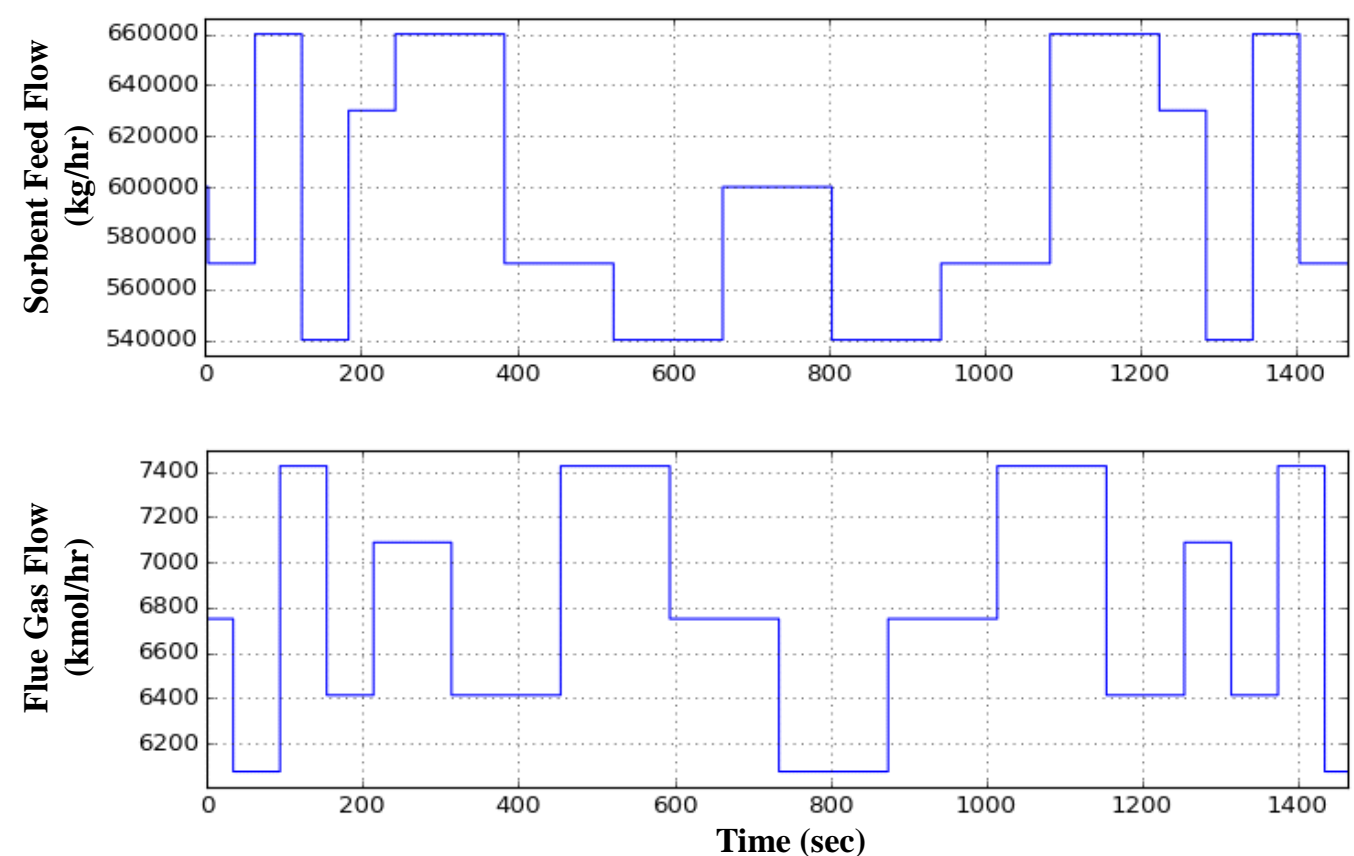

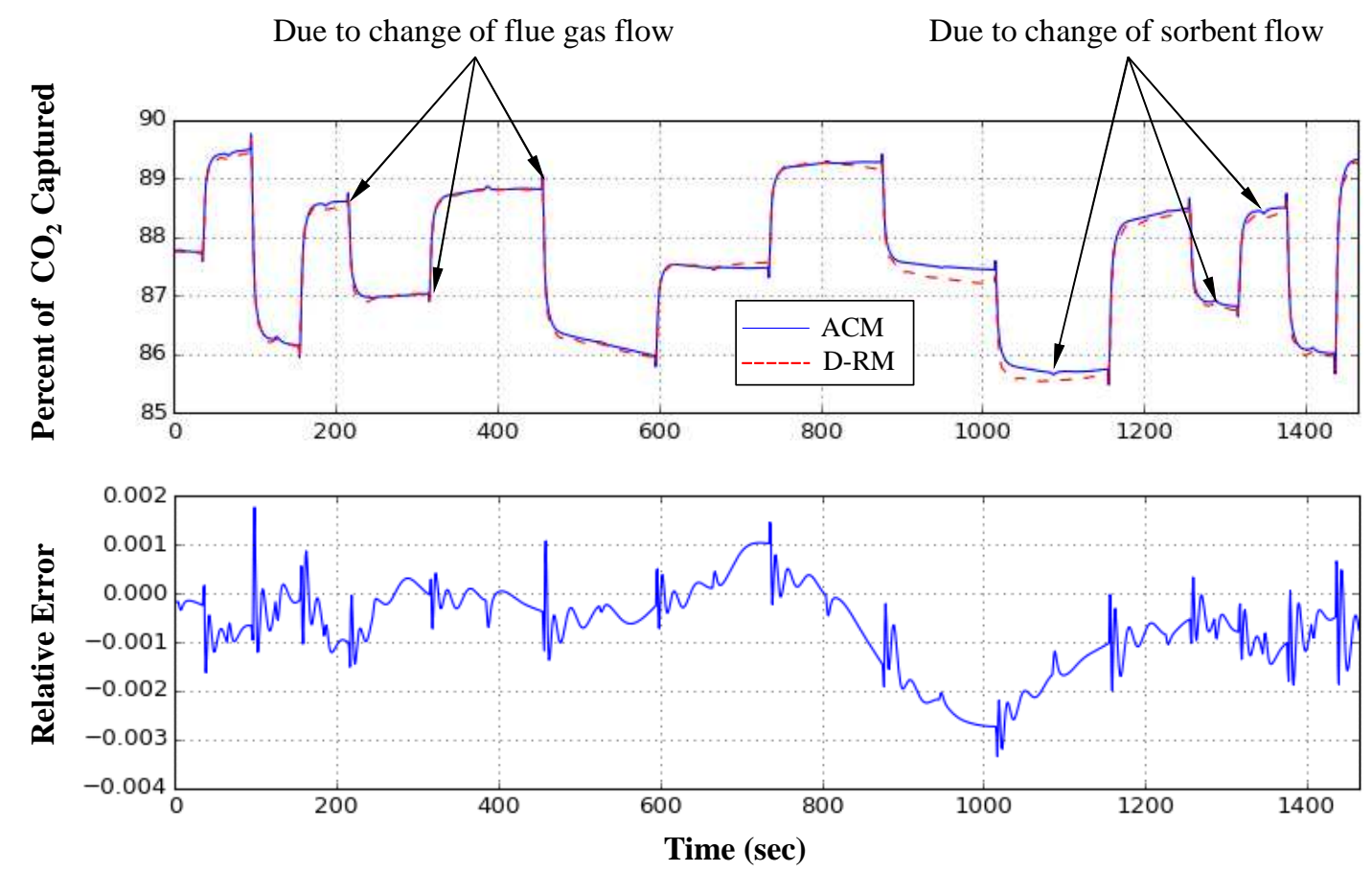

To generate a better D-RM that captures both the fast and slow time scales, the doublepole option is used and both poles for each I/O pair are optimized. Table 6 shows the options and parameters used for training the DABNet model. The Laguerre order for the fast pole is set to 6 , and that for the slow pole is also set to 6 for a total of 12 states for each $\mathrm{I} / \mathrm{O}$ pair. The fast pole is set to 0.5 initially and the slow pole is set to 0.99 initially. Both are optimized by D-RM Builder. It took approximately $600 \mathrm{~s}$ to train the DABNet model and optimize the pole values. At a given set of pole values, the required time for training the DABNet model is approximately $10 \mathrm{~s}$. Table 7 shows the results along with the state vector size of the balanced model. Figure 13 compares the responses of the validation sequence with D-RM results. It took approximately 4 hours for the ACM model to simulate the validation step-change sequence on the Dell workstation while it took less than $1 \mathrm{~s}$ for the D-RM model to predict the response on the same computer. The speedup by using the D-RM is at least four orders of magnitude. It can be seen from Figure 13 that both the fast and slow responses are matched quite well. The average error when training the ANN of the balanced model is $5.10 \times 10^{-5}$ and the $R^{2}$ value for the validation data is 0.99935 as shown in Figure 14, indicating a much more accurate D-RM than the one generated with the single-pole option. 


\begin{tabular}{|l|c|}
\hline \multicolumn{1}{|c|}{ Option } & Selection \\
\hline Model type & DABNet \\
\hline Method for training Laguerre model ANN & BP \\
\hline Method for training balanced model ANN & BP \\
\hline Maximum number of iterations for training Laguerre model ANN & 5000 \\
\hline Maximum number of iterations for training balanced model ANN & 10000 \\
\hline Number of neurons in hidden layer for output $y_{\text {eaptured }}$ & 2 \\
\hline Optimize pole values for output $y_{\text {eaptured }}$ & Yes \\
\hline Time delay applied for I/O pair flow $F / y_{\text {eapturad }}$ & No \\
\hline Number of Laguerre states for I/O pair $F / y_{\text {capturad }}$ & 6 \\
\hline Time delay applied for I/O pair $F_{m} / y_{\text {capturad }}$ & No \\
\hline Number of Laguerre states for I/O pair $F_{m} / y_{\text {capturad }}$ & 6 \\
\hline Initial pole value for all I/O pairs (fast/slow) & $0.5 / 0.99$ \\
\hline
\end{tabular}

Table 7. Optimized pole values and other results for the BFB adsorber-reactor

\begin{tabular}{|l|c|}
\hline Optimized fast pole value for I/O pair $F / y_{\text {eapturad }}$ & 0.575 \\
\hline Optimized slow pole value for I/O pair $F / y_{\text {capturad }}$ & 0.9941 \\
\hline Optimized fast pole value for I/O pair $F_{m} / y_{\text {capturad }}$ & 0.525 \\
\hline Optimized slow pole value for I/O pair $F_{m} / y_{\text {capturad }}$ & 0.9965 \\
\hline Number of states of balanced model for I/O pair $F / y_{\text {capturad }}$ & 10 \\
\hline Number of states of balanced model for I/O pair $F_{m} / y_{\text {eapturad }}$ & 12 \\
\hline Average error of the balanced model ANN for output $y_{\text {capturad }}$ & $5.1 \times 10^{-5}$ \\
\hline
\end{tabular}



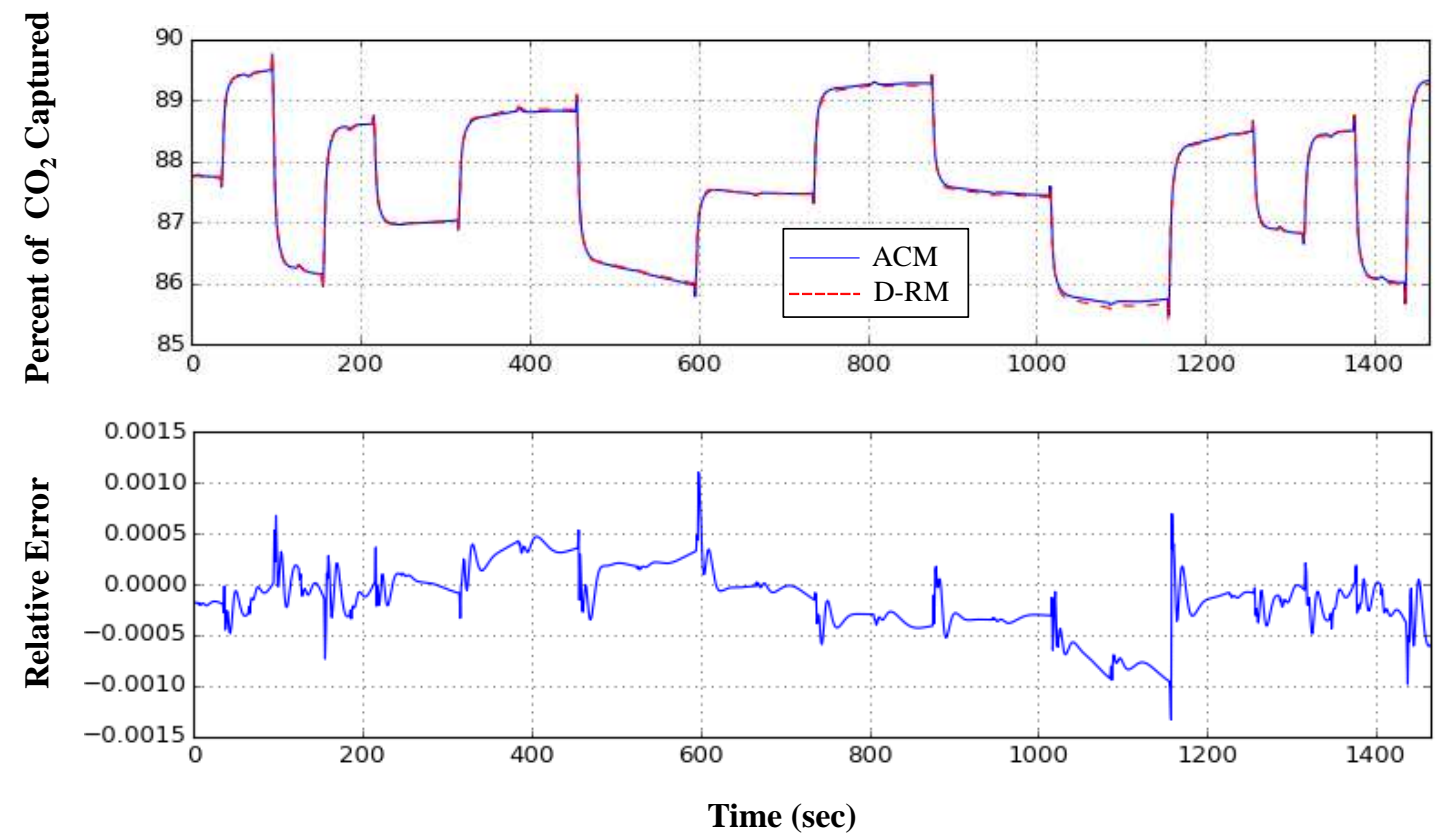

1

4

\section{4. Conclusions and Future Work}

Figure 13. Response to validation sequence predicted by the double-pole DABNet model

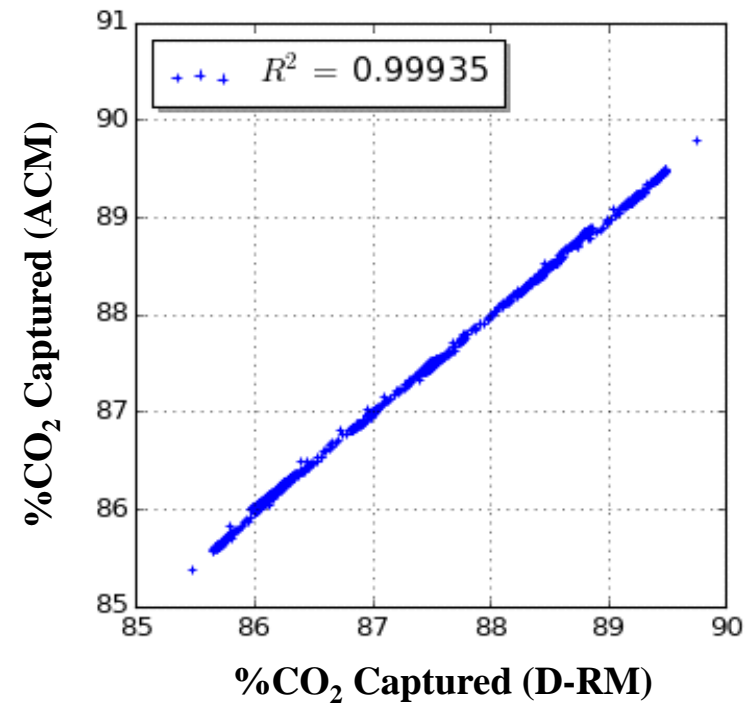

Figure 14. Correlation plot of the percentage of $\mathrm{CO}_{2}$ captured predicted by ACM and D-RM for the validation sequence

A useful software tool, D-RM Builder, was developed to generate fast and accurate datadriven dynamic reduced models (D-RMs) automatically from rigorous dynamic models in Aspen 
Custom Modeler (ACM). The state-space based Decoupled A-B Net (DABNet) model of Sentoni et al. (1998) was chosen as the main nonlinear system identification model to fit the dynamic data generated in response to a series of step changes of input variables in ranges near normal operating conditions with user-defined holding times. Multiple enhancements of the DABNet model have been made including (1) multiple input and multiple output option, (2) double-pole formulation to handle fast/slow time scales, and (3) pole value optimization. Built as a module within the Framework for Optimization, Quantification of Uncertainty and Surrogates (FOQUS) developed under the U.S. Department of Energy's Carbon Capture Simulation Initiative (CCSI), D-RM Builder allows a user to configure the input/output variables, sample input spaces to generate sequences of step changes, launch high-fidelity ACM simulations, fit simulation results to a D-RM, and visualize and validate the resulting D-RM. Once a D-RM is created from the training data, it can be used to predict transient responses and compare them with the corresponding high-fidelity model results. Relative errors and correlation plots can be displayed through the FOQUS GUI. Uncertainty quantification based on unscented Kalman filter can also be performed. The D-RM can be exported as a MATLAB script that works with MATLAB classes developed to perform dynamic simulations and to integrate with the CCSI Advanced Process Control framework. D-RM Builder has been used successfully to generate fast and accurate D-RMs for a highly nonlinear $\mathrm{pH}$ neutralization reactor system and a two-time-scale bubbling fluidized bed adsorber-reactor for $\mathrm{CO}_{2}$ capture. Further improvements are expected to be achieved by optimizing additional model parameters such as the orders of Laguerre series and the number of neurons in the hidden layer of the ANN.

\section{Acknowledgment and Disclaimer}

This work was supported by the U.S. Department of Energy, Office of Fossil Energy as part of the Carbon Capture Simulation Initiative (CCSI). This technical effort was performed in support of the National Energy Technology Laboratory's ongoing research under the RES contract DE-FE0004000. This report was prepared as an account of work sponsored by an agency of the United States Government. Neither the United States Government nor any agency thereof, nor any of their employees, makes any warranty, express or implied, or assumes any legal liability or responsibility for the accuracy, completeness, or usefulness of any information, apparatus, product, or process disclosed, or represents that its use would not infringe privately owned rights. Reference herein to any specific commercial product, process, or service by trade name, trademark, manufacturer, or otherwise does not necessarily constitute or imply its endorsement, recommendation, or favoring by the United States Government or any agency thereof. The views and opinions of authors expressed herein do not necessarily state or reflect those of the United States Government or any agency thereof.

\section{Nomenclature}

\section{Symbols}

$38 a_{i j} \quad$ pole value of Laguerre series for $i$ th output and $j$ th input 


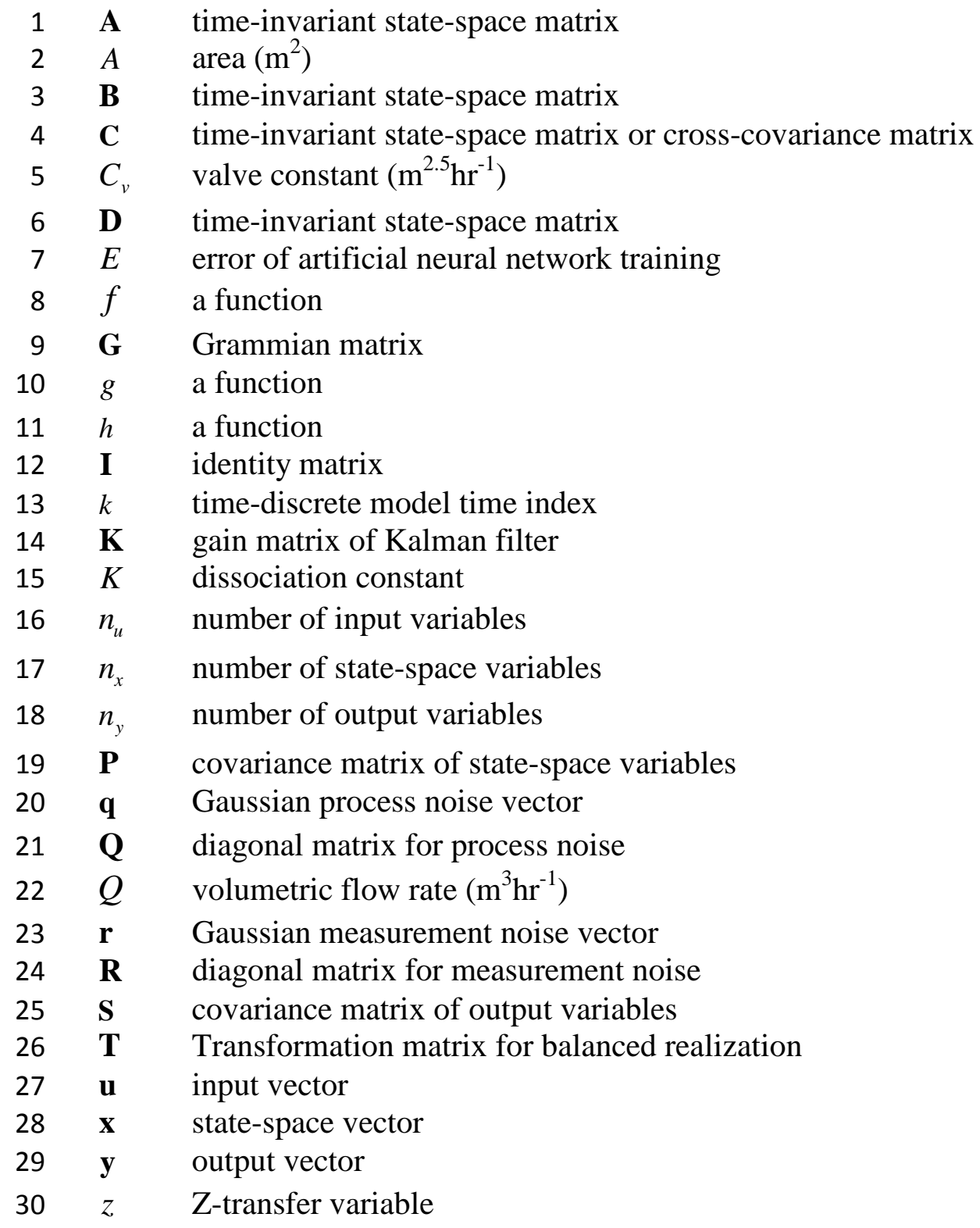

\section{Greek letters}

$33 \alpha \quad$ acetate $\left(\mathrm{HAC}+\mathrm{AC}^{-}\right)$concentration $\left(\mathrm{kmol} \mathrm{m}^{-3}\right)$

$34 \beta \quad$ sodium $\left(\mathrm{N}_{\mathrm{a}}^{+}\right)$concentration $\left(\mathrm{kmol} \mathrm{m}^{-3}\right)$

$35 \Delta t \quad$ sampling time interval (s)

$36 \quad \sigma \quad$ standard deviation

$37 \tau_{i j} \quad$ time constant for $i$ th output and $j$ th input (s)

38

\section{Subscripts}

40 bal balanced

$41 \quad c \quad$ controllability 


$\begin{array}{rll}1 & f & \text { fast } \\ 2 & i & \text { output variable index } \\ 3 & j & \text { input variable index } \\ 4 & o & \text { obserbability } \\ 5 & r & \text { term index for truncation } \\ 6 & s & \text { slow } \\ 7 & t & \text { target } \\ 8 & & \\ 9 & \text { Superscripts } \\ 10 & - & \text { mean } \\ 11 & \text { aug } & \text { augmented } \\ 12 & \text { ANN } & \text { artificial neural network } \\ 13 & & \end{array}$

\section{References}

Agarwal, K., P. Sharma, J. Ma, C. Lo, I. Gorton, and Y. Liu, "Reveal: An Extensible ReducedOrder Model Builder for Simulation and Modeling," Computing in Science \& Engineering, Vol. 16, No. 21, 44-53 (2014).

Cozad, A., N. Sahinidis, and D. C. Miller, "Learning Surrogate Models for Simulation-Based Optimization." AIChE Journal. 60, 2211-2227 (2014).

Eslick, J. C., B. Ng, Q. Gao, C. H. Tong, N. V. Sahinidis, and D. C. Miller, "A Framework for Optimization and Quantification of Uncertainty and Sensitivity for Developing Carbon Capture Systems," Energy Procedia, 63, 1055-1063 (2014).

Lee, A., and D. C. Miller, "A One-Dimensional (1-D) Three-Region Model for a Bubbling Fluidized-Bed Adsorber," Industrial \& Engineering Chemistry Research, 52, 469-484 (2013).

Hartikainen, J, Solin, A and Sarkka, S 2011, "Optimal Filtering with Kalman Filters and Smoothers, a Manual for the Matlab toolbox EKF/UKF”, version 1.3, Aalto University School of Science, Espoo, Finland.

Mahapatra, P., J. Ma, B. Ng, D. Bhattacharyya, S. E. Zitney, and D. C. Miller, "Integrated Dynamic Modeling and Advanced Process Control of Carbon Capture Systems," Energy Procedia, 63, 1354-1367 (2014).

Mathworks, Inc., "System Identification Toolbox," MATLAB documentation, available at http://www.mathworks.com/products/sysid/ (2015). 
Dale, "Carbon Capture Simulation Initiative: A Case Study in Multiscale Modeling and New Challenges," Annual Review of Chemical and Biomolecular Engineering, 5, 301-323 (2014a).

Miller, D. C., B. Ng, J. Eslick, C. Tong, and Y. Chen, "Advanced Computational Tools for Optimization and Uncertainty Quantification of Carbon Capture Process," Proceedings of the $8^{\text {th }}$ International Conference on Foundations of Computer-Aided Process Design, July 13-17, Cle Elum, Washington, USA (2014b).

Miller, D. C., X. Sun, C. B. Storlie, and D. Bhattacharyya, "Using Advanced Modeling to Accelerate the Scale-Up of Carbon Capture Technologies," Power Engineering, 119 (2015).

Mitsis, G. D., and V. Z. Marmarelis, "Modeling of Nonlinear Physiological Systems with Fast and Slow Dynamics. I. Methodology," Annals of Biomedical Engineering, Vol. 30, 272-281 (2002).

Modekurti, S., D. Bhattacharyya, and S. E. Zitney, "Dynamic Modeling and Control Studies of a Two-Stage Bubbling Fluidized Bed Adsorber-Reactor for Solid-Sorbent $\mathrm{CO}_{2}$ Capture," Industrial \& Engineering Chemistry Research, 52, 10250-10260 (2013).

Nelder, J. A., and R. Mead, "A simplex method for function minimization," Computer Journal, Vol. 7, pp.308-313 (1965).

Narendra, K., and Mukhopadhyay, S., "Adaptive control using neural networks and approximate models," IEEE Transactions on Neural Networks, vol. 8, No. 3, 475-485 (1997).

PySide Project, "PySide Documentation," available at https://wiki.qt.io/PySideDocumentation (2015).

Riedmiller, M., "Rprop - Description and Implementation Details," Technical report (1994), available at http://www.inf.fu-berlin.de/lehre/Ws06/Musterererkennung/Paper/rprop.pdf

Sentoni, G. B., Biegler, L. T., Guiver, J. B., and Zhao, H.; "State-space nonlinear process modeling: identification and universality," AIChE Journal, vol. 44, No. 10, 2229-2239 (1998).

Sjöberg, J, Q. Zhang, L. Ljung, A. Benveniste, B. Delyon, P. Glorennec, H. Hjalmarsson, A. Juditsky, "Nonlinear black-box modeling in system identification: a unified overview," Automatica, 31, 1691-1724 (1995).

The Qt Company Ltd., Qt Document Website at http://doc.qt.io/qt-4.8/designer-manual.html (2015).

Tsoi, A. C., and A. D. Back, "Locally Recurrent Globally Feedforward Networks: A Critical Review of Architectures," IEEE Trans. On Neural Networks, 5(2), 229 (1994). 
1 Wächter, A. and L. T. Biegler, "On the Implementation of a Primal-Dual Interior Point Filter 2 Line Search Algorithm for Large-Scale Nonlinear Programming", Mathematical Programming, 3 vol. 106, no. 1, 25-57 (2006).

Yu, M., D. C. Miller, and L. T. Biegler, "Dynamic Reduced Order Models for Simulating Bubbling Fluidized Bed Adsorbers," Industrial \& Engineering Chemistry Research, 54, 69596974 (2015).

Zervos, C., P. Belanger, and G. Dumont, "On PID Controller Tuning using Orthonormal Series Identification," Automatica, 24, 165 (1988).

Zhou, K., J. C. Doyle, and K. Glover, "Robust and Optimal Control,” Prentice Hall, Englewood 14

- A software tool is developed to generate dynamic reduced models from high-fidelity models

- A nonlinear system identification model is enhanced to handle slow and fast time scales

- Metrics are developed to measure the accuracy of generated dynamic reduced models

- A fully integrated software tool has been tested on simple benchmark nonlinear systems and complicated $\mathrm{CO}_{2}$ capture systems 